\title{
Die Nesselkapseln der Anthozoen und ihre Bedeutung für die phylogenetische Systematik
}

\author{
H. SCHMIDT \\ Zoologisches Institut der Universität Heidelberg (Morphologischer Lebrstubl); \\ Heidelberg, Bundesrepublik Deutschland
}

\begin{abstract}
The nematocysts of the anthozoans and their importance for phylogenetical systematics. Nematocysts of 35 anthozoan species from Atlantic Ocean, Mediterranean Sea and Red Sea were studied employing phase contrast and electron microscopy. The nematocyst-types of WeILL's (1934) system exhibit a great divergence between different anthozoan orders. Therefore, they are of little taxonomic and phylogenetical value. Atrichous haplonemes occur in the Ceriantharia and in the Actiniaria; all other Hexacorallia have holotrichous haplonemes with very different spines. Sometimes they are morphologically much more differentiated than the spines of the rhabdoid heteronemes, the only nematocyst-type present in the Octocorallia. If their distal ends form a $T$, the spines are considered plesiomorphous; they are present on the tube of some holotrichous haplonemes as well as on the thread of some rhabdoid heteronemes. CARLGREN's (1940) subdivision of the rhabdoid heteronemes is quite useful because the b-and p-rhabdoid differ in all orders of Hexacorallia by having a different armature on the thread. Ceriantharia are considered to represent the most plesiomorphous group among the Hexacorallia. They have the simplest haplonemes (tube atrichous ore with spines forming a $T$ ) and rhabdoids heteronemes with the most differentiated shaft, but feature plesiomorphous $T$-forming spines on the thread of the b-rhabdoids. In certain Ceriantharia, p-rhabdoids occur with a short anoplotelic thread which is synapomorphous in the Ceriantharia as well as in the Actiniaria and Zoantharia. The rather differentiated shaft is a symplesiomorphous character of the Ceriantharia and the "early" Actiniaria, which exhibit other symplesiomorphous characters, ectodermal longitudinal muscles in the scapus and the same swimming behaviour (Robson 1966). The Zoantharia are derived from the "late" Actiniaria. Plesiomorphous Zoantharia, like Endomyaria of the Actiniaria, possess large b-rhabdoids in their mesenterial filaments. In addition, all Zoantharia have exactly the same p-rhabdoids, common in the Endomyaria; p-rhabdoids differ only in the Antipatharia in that they have a somewhat longer thread, which are armed with single spines. The Endomyaria, as well as the Zoantharia and Antipatharia, are also synapomorphous in the main distribution of their $\mathrm{b}$-rhabdoids and in having a special form of sperm. In addition, Antipatharia have the same normal b-rhabdoids as the Zoantharia and the same chemical composition of the skeleton as the skeleton-forming Zoantharia Gerardia savaglia, which differs markedly from the chemical composition of the gorgonian skeleton (Roche \& TrXIER-Durivault 1951).
\end{abstract}

\section{EINLEITUNG}

Die phylogenetisch-systematischen Forschungen über Anthozoen sind bisher nicht über das spekulative Stadium hinausgekommen. Bei phylogenetischen Erörterungen 
haben die Nesselkapseln - die wichtigsten Merkmale der Anthozoen wie der übrigen Cnidaria - eine bemerkenswert geringe, oft überhaupt keine Rolle gespielt. Der Grund lag einerseits darin, daß die Nesselkapseln vieler Anthozoengruppen nur sehr mangelhaft bekannt sind. Andererseits mußten die zumeist älteren lichtmikroskopischen Arbeiten (u. a. Seifert 1928, Stephenson 1929, Weill 1934, Tischbierek 1936, CarlGren 1940, 1945, Cutress 1955, Hand 1961, Werner 1965) den feineren Bau der Nesselkapseln unberücksichtigt lassen. Dieser Umstand führte zu mehr oder weniger willkürlich errichteten Nesselkapseltypen, deren Homologie bei den einzelnen Anthozoen keineswegs bewiesen war.

WeSTFALL $(1962,1965)$ konnte erstmals anhand elektronenoptischer Studien den grundsätzlichen Unterschied zwischen den Nesselkapseln der Hydrozoen und Scyphozoen einerseits und den Anthozoen andererseits darstellen. Mit der vorliegenden Arbeit soll damit begonnen werden, die Ultrastruktur der Nesselkapseln der Anthozoen zu untersuchen, um sie später besser als konstitutive Merkmale der phylogenetischen Systematik (Hennig 1969) verwenden zu können. In diesem Sinne wurden bereits die Nesselkapseln der Actiniaria typenmäßig neu gegliedert (Sснмпт 1969). Dadurch war es erstmals möglich, die Nesselkapseln der Actiniaria als konstitutive Merkmale zu verwenden, das heißt, ihre diagnostische Bedeutung durch Vergleich mit anderen konstitutiven Merkmalen zu belegen und die phylogenetische Entwicklung innerhalb der Actiniaria aufzuzeigen (SсHмrd 1972b). In der vorliegenden Arbeit werden die Nesselkapseln von Vertretern fast aller Anthozoenordnungen (vgl. KAEsTNER 1969), mit Ausnahme der Helioporida, untersucht und die diagnostische Bedeutung kurz aufgezeigt. Die Einteilung der verschiedenen Nesselkapseltypen ist nur als vorläufige Klassifizierung zu betrachten.

\section{MATERIAL, METHODE UND TERMINOLOGIE}

Nesselkapseln von 35 verschiedenen Anthozoenarten aus dem Atlantik, dem Mittelmeer und dem Roten Meer wurden in lebensfrischem Zustand im Phasenkontrast untersucht. Die Entladung der meisten Kapseln wurde durch Zusatz von Aqua destillata oder 1- bis 10\% igem Eisessig ausgelöst. Die elektronenoptischen Untersuchungen fanden ausschließlich an Totalpräparaten statt (vgl. Robson 1953, SCHMidT 1969). Zur Negativkontrastierung wurden die Trockenpräparate mit 4\% oiger Phosphorwolframsäure mit einem Zusatz von 0,4\% iger Sacharose bei einem pH-Wert von 7 etwa eine bis drei Minuten behandelt. Die Terminologie richtet sich nach dem von CARLGREN (1940) und Schmidt $(1969,1972 b)$ abgeänderten WeIllschen System (Wexll 1934, WERNER 1965).

Danach werden zur Zeit bei den Anthozoen folgende Nesselkapseltypen unterschieden:

(I) Isorhize oder anisorhize holotriche oder atriche Haplonemen : Nesselkapseln mit weitgehend isodiametrischem oder anisodiametrischem, unbewaffnetem oder bewaffnetem einheitlichem Schlauch, ohne abgesetzten proximalen Schaft oder abgesetzte Bewaffnung.

(II) Rhabdoide He te r o n e m e n Nesselkapseln mit deutlich abgesetzter Be- 
waffnung am proximalen Schlauch, der daher in Schaft and Faden unterteilbar ist. Der Schaft ist bei den rhabdoiden Heteronemen weitgehend isodiametrisch und stabförmig.

Nach der Bewaffnung des Endfadens werden hoplotele (bewaffnet) und anoplotele (unbewaffnet) Rhabdoiden unterschieden. CARLGREN (1940) unterteilte die Rhabdoiden der Anthozoen in p-Rhabdoiden (nicht entladener Schatt am distalen Ende mit trichterförmiger Ơffnung) und b-Rhabdoiden (Schaft ohne trichterförmige Offnung).

\section{ERGEBNISSE}

Die Nesselkapseln der Anthozoen bestehen aus Spirocysten und Nematocysten (Nesselkapseln im eigentlichen Sinn). Spirocysten kommen nur bei den Hexacorallia vor und zeigen bei den einzelnen Ordnungen keine wesentlichen Unterschiede. Sie werden daher im speziellen Teil nicht weiter berücksichtigt.

Wie bei den phylogenetisch älteren Actiniaria bleibt der Quellfaden auch bei den Spirocysten der Ceriantharia häufig eine kurze Zeit unmittelbar nach der Explosion erhalten und ist in diesem Zustand relativ leicht im Lichtmikroskop zu beobachten (Schmid 1969, Abb. 1a, b). Die Explosion der Spirocysten und die Funktion des Quellfadens sind seit langem bekannt (Will 1909, vgl. Stephenson 1929, Schmor 1969). Die jüngsten elektronenoptischen Untersuchungen von Doumenc (1971) zu diesem Thema sind allerdings irreführend. Doumenc konnte den Quellfaden und die nach dessen Zerfall erfolgende Ausbreitung der Klebmasse nach der Einwirkung von Fixierungsgemischen selbstverständlich nicht beobachten, da die Quellsubstanz koagulierte. Zuvor hatten schon SkAer \& PICKEN (1965, Abb. 25b) die Ausbreitung der Quellsubstanz im Elektronenmikroskop dargestellt.

Spirocysten und Nematocysten unterscheiden sich außerdem in der inneren Kapselstruktur. Die Kapseln der Spirocysten sind im Gegensatz zu den Nematocysten innen mit regelmäßigen Falten ausgekleidet (Westfall 1965, Skaer \& Picken 1965; DouMENC 1971). Die Spirocysten und der größte Teil der Nematocysten stimmen dagegen in der äußeren Kapselstruktur überein. Sie besteht aus schrägverlaufenden Fibrillen (Skaer \& PiCKen 1965, Doumenc 1971), die sich im. Negativkontrast gut abheben (Abb. 1d, 8f), aber bei starker Vergrößerung auch ohne Kontrastierung zu erkennen sind (Abb. 3a, c). Die Fibrillen verlaufen bis zur Kapselöffnung, und zwar stets schräg in Linkswindungen (vgl. Doumenc 1971). Für die vorliegende Untersuchung sind folgende Befunde von Bedeutung:

Das Fibrillenmuster ist bei den Spirocysten und den Nematocysten der Octocorallia, der Madreporaria und der Ceriantharia regelmäßig, da die Fibrillen ziemlich parallel laufen (Abb. 1d). Bei den p-Rhabdoiden A der Aktinien und den p-Rhabdoiden der Zoantharien und Antipatharien ist der Abstand zwischen den Fibrillen sehr wechselnd, wodurch sich ein unregelmäßiges Fibrillenmuster ergibt (Abb. 8f). Im Gegensatz zu den p-Rhabdoiden A und den Nematocysten und Spirocysten der übrigen Anthozoen sind bei allen anderen Rhabdoiden der Aktinien keine Fibrillen mit Hilfe der Negativkontrastierung darstellbar. Mit Ausnahme der p-Rhabdoiden A unterscheiden sich die Rhabdoiden der Aktinien von den Nesselkapseln der übrigen Anthozoen durch 
die relativ dicken Kapselwände und die auffälligen Offnungsklappen (vgl. SkaER \& Picken 1965, Westfall. 1965, Schmidt 1969).

\section{Octocorallia}

Die Nesselkapseln der Octocorallia fanden in der bisherigen Literatur wegen ihrer geringen Größe nur wenig Beachtung. Im Lichtmikroskop sind selten mehr als ein einfacher Schlauch und die Kapsel zu unterscheiden. Daher wurden sie von WeILL (1934) als atriche Haplonemen bezeichnet. An dem ausgestülpten Schlauch der Nesselkapseln der Alcyonarien und Gorgonarien ist aber im Phasenkontrast deutlich ein dicker Anfangsteil (Schaft) von einem dünneren Endteil (Faden) zu unterscheiden. An den Nesselkapseln einiger Alcyonarien ist sogar die Bewaffnung des Schaftes zu erkennen. Ahnliche Beobachtungen machte auch Cutress (1955), der die Nesselkapseln der Octocorallia daher als basitriche Haplonemen bezeichnete.

Die elektronenoptischen Befunde (Abb. 1) zeigen, daß es sich bei den Nesselkapseln der Octocorallia um eindeutige rhabdoide Heteronemen handelt. Sie sind als solche an der deutlich abgesetzten Bewaffnung des Schaftes zu erkennen. Der Heteronemencharakter kommt auch darin zum Ausdruck, daß Schaft und Faden unterschiedliche Dornen aufweisen (Abb. 1b, c, f, g). Der Schaft ist in explodiertem Zustand stets länger als die Kapsel (Abb. 1a) und erreicht häufig die zwei- bis fünffache Kapsellänge. Die Dornen (Abb. 1b, e, f) haben eine breite Basis, ein gerades Mittelstück und sind am Ende zugespitzt. Sie sind in undeutlichen Windungen angeordnet. An den Schaft schließt sich ein weniger dicht bewaffneter Faden von unterschiedlicher Länge an. Seine Dornen sind spitz oder laufen am distalen Ende unregelmäßig T-förmig in zwei Spitzen aus, wobei eine Spitze bedeutend länger ist als die andere (Abb. $1 \mathrm{c})$. Die große Länge des Schaftes bedingt, daß auch dieser Schlauchteil in mehreren Schrägwindungen in der geschlossenen Kapsel aufgerollt ist. Da außerdem in der geschlossenen Kapsel der Übergang zwischen Schaft und Faden nicht feststellbar ist, ist der Heteronemencharakter der Nesselkapseln der Octocorallia im Lichtmikroskop in unentladenem Zustand nicht zu erkennen. Die äußere Form der Kapsel ist häufig bei den verschiedenen taxonomischen Kategorien recht unterschiedlich. Schon WEILL (1934) hat erkannt, daß die Nesselkapseln vieler Octocorallia der äußeren Form nach in zwei verschiedenen Formen vorkommen. Bei den hier untersuchten Arten unterscheiden sich beide Formen mehr oder weniger deutlich wie folgt:

(I) Häufig mehr rundliche Kapselform, in geschlossenem Zustand deutlicher Phasenkontrast, so daß der Schlauch nur schwer zu erkennen ist. Der Schlauch ist relativ kurz und füllt nur etwa die Hälfte der Kapsel aus. Die Schaftbewaffnung ist aufgelockert (Abb. 1e) und setzt sich daher weniger gut von dem kräftig bedornten Faden ab. Dornen des Fadens mehr oder weniger $T$-förmig (Abb. 1c).

(II) Kapsel meist mehr schlank als rundlich, Inhalt zeigt kaum Phasenkontrast, wodurch der lange, die ganze Kapsel ausfüllende Schlauch in unentladenem Zustand besonders gut erkennbar ist. Schaftbewaffnung dicht (Abb. 1b, f), Faden spärlich bewaffnet und daher gut abgesetzt (Abb. 1a), Dornen spitz (Abb. 1g).

Vermutlich wurde Form II des in der Kapsel deutlich sichtbaren Schlauches wegen 

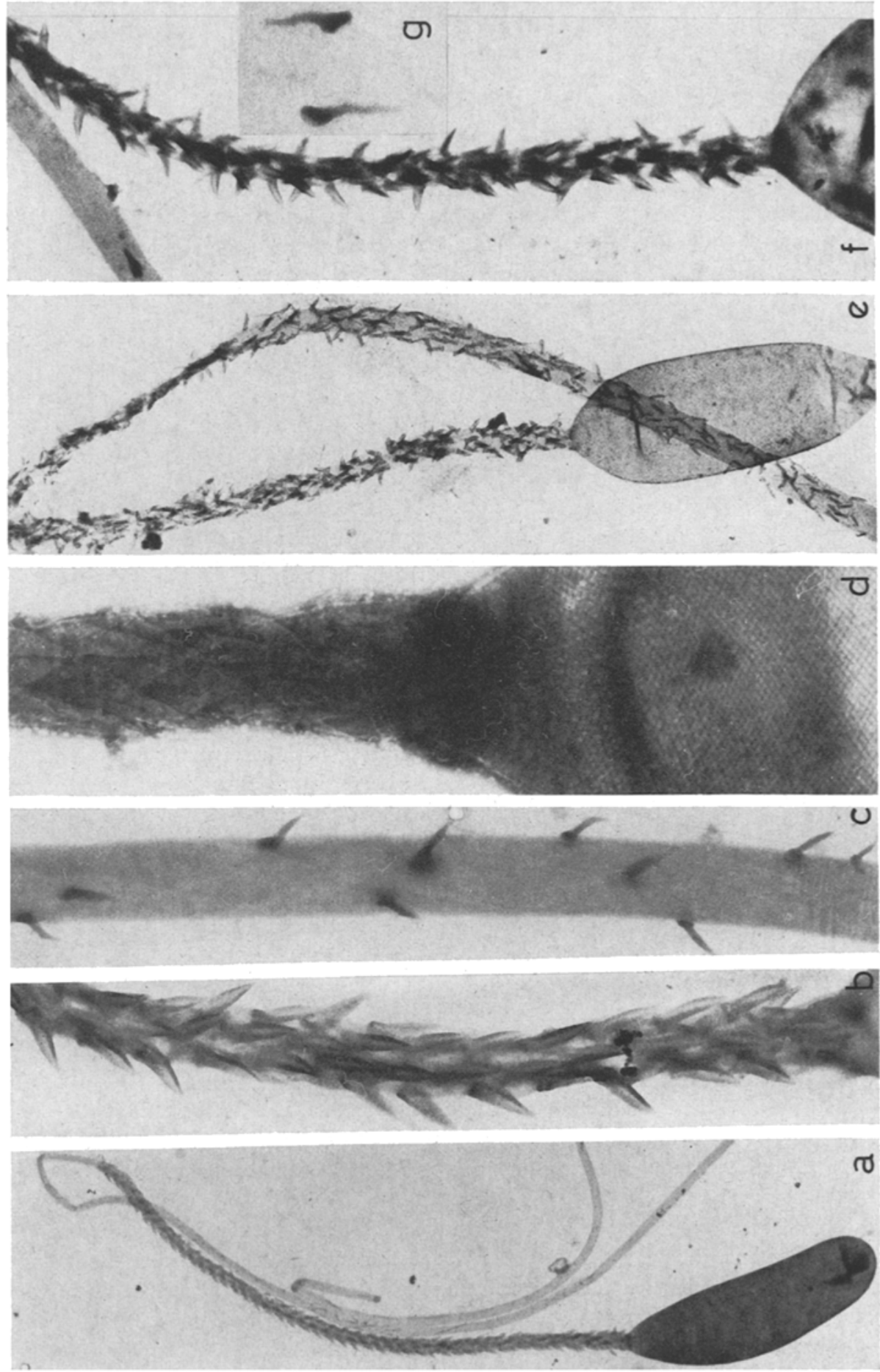
von früheren Autoren (vgl. WEILL 1934) für Spirocysten gehalten, da sie außerdem nur im Ektoderm der Tentakeln und - weniger häufig - der Mundscheibe vorkommt. Bei Alcyonium palmatum und bei Parerythropodium coralloides sind beide Varietäten form- und größengleich, so daß sie schwer zu unterscheiden sind. Bei Heteroxenia fuscescens z. B. sind beide Formen leicht zu erkennen. Bei Pennatula rubra (Pennatulidae) und bei Pteroeides spinosum (Pteroeididae) ist Form I bedeutend größer als Form II. Bei Paralcyonium elegans konnte ich Form I nicht eindeutig nachweisen. Form II ist im allgemeinen viel häufiger als Form I, die sehr selten auch im Pharynx und in den Filamenten vorkommen kann.

Die vorliegenden Befunde lassen erkennen, daß die Octocorallia zwar nur über ein Monocnidom (WeILl 1934, vgl. WERNER 1965) verfügen, daß sich aber die verschieden Kapselformen und -größen taxonomisch verwerten lassen (vgl. WErLL 1934).

\section{Hexacorallia}

Im Gegensatz zu den Octocorallia zeichnen sich die Nesselkapseln der Hexacorallia durch eine größere morphologische Mannigfaltigkeit aus. Das Cnidom der Hexacorallia setzt sich allgemein aus Spirocysten, die hier nicht weiter erwähnt werden, und Nematocysten in Form von verschiedenartigen Haplonemen und rhabdoiden Heteronemen zusammen. Die einzelnen Ordnungen sind daher separat zu behandeln.

\section{Ceriantharia}

Die Taxonomie der Ceriantharien befindet sich zur Zeit in einem ebenso chaotischen Zustand wie die Kategorisierung ihrer Nesselkapseln, insbesondere der Heteronemen. Für die Untersuchung standen 4 Arten zur Verfügung: Cerianthus membranaceus, Cerianthus maua, Pachycerianthus multiplicatus und eine Ceriantboides nov. gen. nov. spec. Alle Arten unterscheiden sich deutlich in ihrem Cnidom.

\section{Die gegenwärtige Klassifizierung}

WEILL (1934) hat zwar keine eigenen Untersuchungen an Ceriantharien vorgenommen; er faßt aber die derzeitigen Erkenntnisse über die Nesselkapseln der Ceriantharien zusammen und klassifiziert sie nach seinem System. Danach besteht das Cnidom

Abb. 1: $a$ Rhabdoide Heteroneme II, Zoantha rosea (Alcyonaria), Tentakel (3610:1); $b$ rhabdoide Heteroneme II, Zoantha rosea (Alcyonaria), Tentakel : unterer Schafteil (21 160:1); $c$ rhabdoide Heteroneme II, Zoantha rosea (Alcyonaria), Tentakel : Faden (32 870:1); $d$ rhabdoide Heteroneme II, Zoantha rosea (Alcyonaria), Tentakel : Negativkontrast (40000:1); $e$ rhabdoide Heteroneme I, Corallium rubrum (Gorgonaria), Tentakel (7700:1); $f$ rhabdoide Heteroneme II, Veretillum cynomorium (Pennatularia), Tentakel (11440:1); g rhabdoide Heteroneme II, Veretillum cynomorium (Pennatularia), Tentakel : Dornen des Fadens (28 $800: 1)$ 

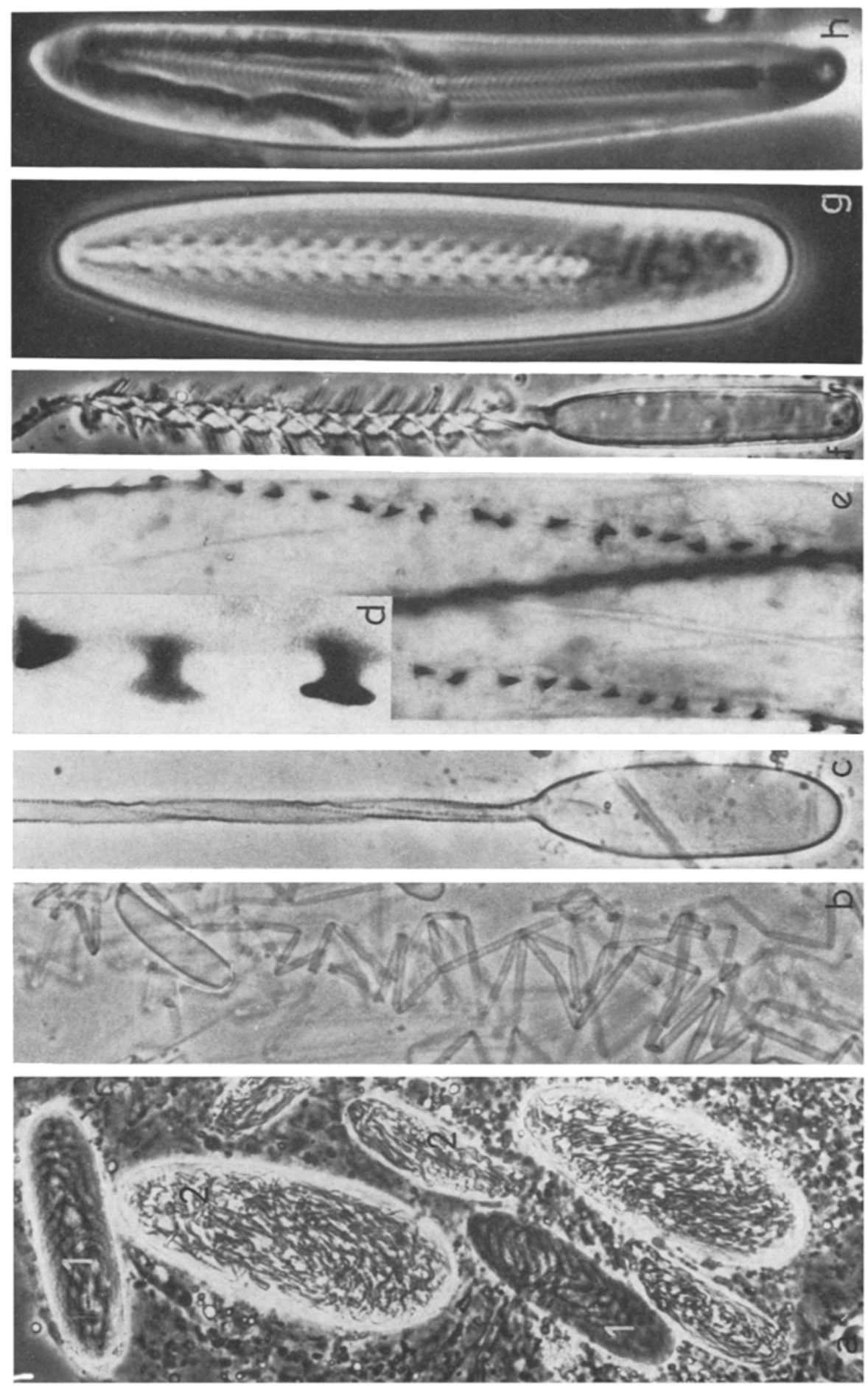
der Ceriantharien aus Spirocysten, atrichen und holotrichen Haplonemen und rhabdoiden Heteronemen in Form von "mikrobasischen Mastigophoren " (Schaft $=3 \times$ Kapsellänge, Faden stets ausgestülpt und mit Schaft verbunden), CARLgreN (1940) hat das Cnidom von Vertretern fast aller derzeit bekannten Familien und Gattungen untersucht und Wenls Angaben bestätigt. Nach seiner Auffassung handelt es sich bei den "mikrobasischen Mastigophoren“ der Ceriantharia ausschließlich um b- „Mastigophoren" (= b-Rhabdoiden). Cutress (1955) fand dagegen erstmals bei einem nicht näher bezeichneten Cerianthus p-Rhabdoiden. Der Schaft war vier- bis sechsmal so lang wie die Kapsel und hatte ein unbewaffnetes Faltstück (vgl. ScHмm , 1969) von etwa $2 / 3$ der gesamten Schaftlänge. Nach Cutress (1955, Fig. 8f, g) sollen Faltstück und Faden bewaffnet sein und die Dornen des Faltstückes nach der Explosion abfallen. Auf Grund der trichterförmigen Offnung am distalen Ende des unentladenen Schaftes und der Länge des ausgestullpten Schaftes bildete CuTress (1955) die neue Kategorie der „makrobasischen p-Mastigophoren". Die Kategorie entspricht jedoch nicht der gegebenen Definition (vgl. Westall 1965, Schmot 1969) und umfaßt zum Teil Nesselkapseln, die sich nicht im entferntesten mit den $\mathrm{p}$-Rhabdoiden der Ceriantharia vergleichen lassen, wie u. a. die großen Holotrichen Haplonemen I der Corallimorpharia (vgl. Hand 1961, Skaer \& Picken 1965).

\section{Die Haplonemen}

(1) Atriche Haplonemen: Die Untersuchungen haben ergeben, daß die von WeILL (1934) als atriche Haplonemen charakterisierten Nesselkapseln bei den Ceriantharia tatsächlich atrich sind, d. h. keinerlei Dornen aufweisen.

Der Schlauch der Atrichen ist in der geschlossenen Kapsel wenig dicht gefaltet und daher in zahlreichen unregelmäßigen Windungen angeordnet (Abb. 2a). Der ausgestülpte Schlatich ist glattwandig (Abb, 2b). Atriche können in verschiedenen Größen mehr oder weniger in allen Körperbereichen vorkommen. Sie sind äußerst zahlreich im Ektoderm des Scapus. Ihre ausgestullpten Schläuche bilden den Hauptbestandteil der Wohnröhren (Abb. 2b).

(2) Holotriche Haplonemen kommen ebenfalls vorwiegend im Mauerblatt vor. CARLGREN (1940) fand sie außerdem bei wenigen Arten in den Labialtentakeln und im Pharynx. Die Holotrichen unterscheiden sich von den Atrichen deutlich im Phasenkontrast (Abb. 2a). Beide Nesselkapseltypen sind daher leicht zu diagnostizieren. In explodiertem Zustand ist die Bewaffnung der Holotrichen im Lichtmikroskop deutlich zu erkennen (Abb. 2c). Die Dornen stehen in langgezogenen Spiralen (Abb. 2c, e). Sie

Abb. 2: a Holotriche (1) und atriche (2) Haplonemen, Ceriantbus membranaceus, Scapus $(780: 1) ; b$ atriche Haplonemen, Ceriantbus membranacens, Scapus $(690: 1) ; c$ holotriche Taploneme, Ceriantbus membranaceus, Scapus (810:1); $d$ holotriche Haploneme, Ceriantbus membranaceus, Scapus : Dornen (30500:1); e holotriche Haploneme, Cerianthus membranaceus, Scapus : Schlauch (7500:1); f große b-Rhabdoide I, Cerianthus membranaceus, Mesenterialflament (mittlerer Teil) $(810: 1)$; g große b-Rhabdoide 1 , Ceriantbus membranaceus, Mesenterialfilament (unterer Abschnitt) $(1455: 1)$; $h$ p-Rhabdoide IIb, Ceriantboides spec., Labialtentakel $(1880: 1)$ 

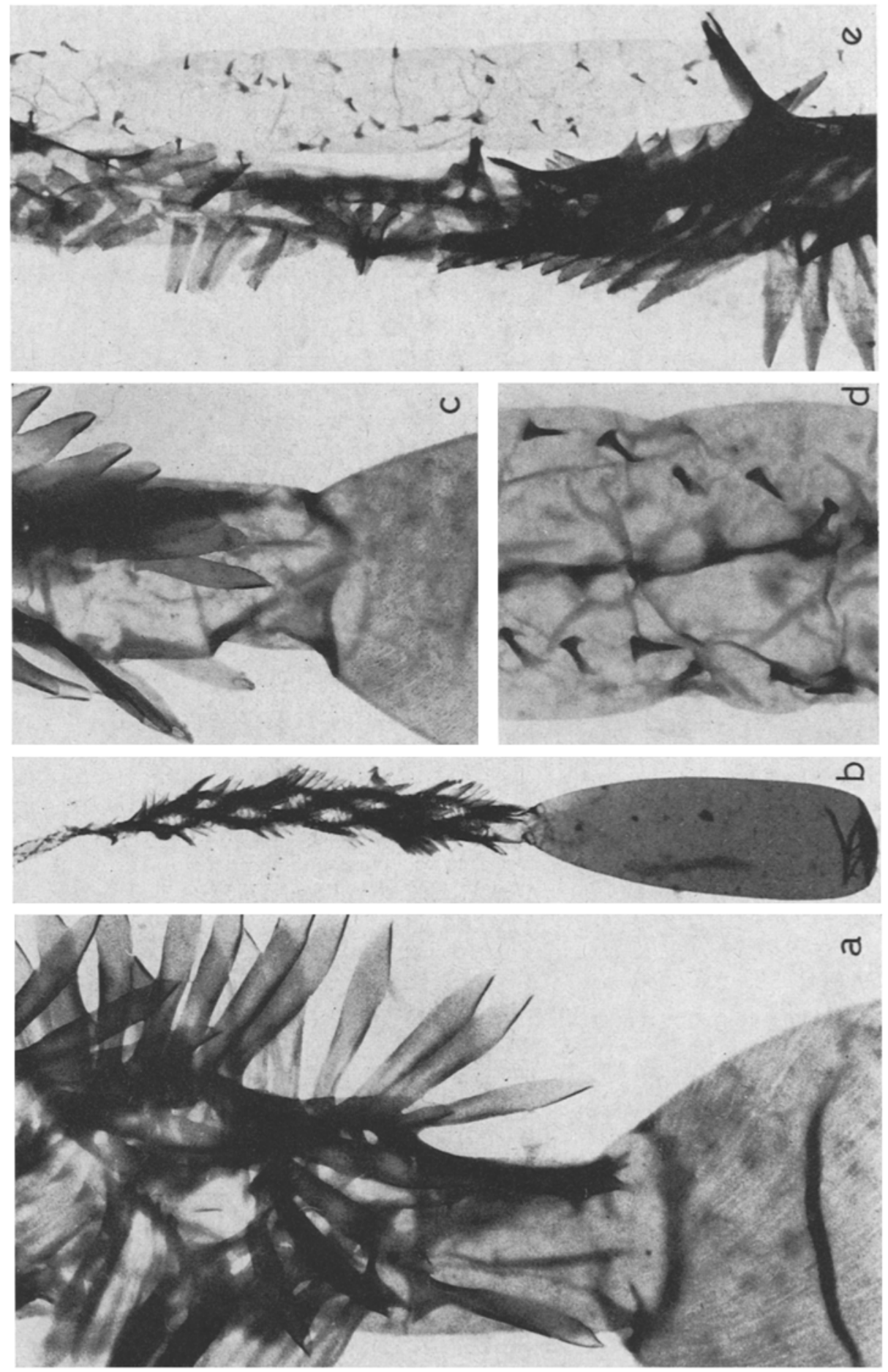
sind relativ klein, bestehen aus ziemlich elektronendichtem Material und sind T-förmig (Abb. 2d, e).

\section{Die Heteronemen}

Bei den Ceriantharien kommen Heteronemen mit oder ohne trichterförmige Einstülpung am unentladenen Schaft vor. Sie sind somit nach CARLGREN (1940) als b- und p-Rhabdoiden zu unterscheiden. Im Gegensatz zu CuTress (1955) besteht aber der Hauptunterschied $z$ wischen beiden Kategorien nicht nur in dem unterschiedlich gestalteten Schaftende, sondern auch in der Länge, Entladung und Bewaffnung des Fadens. Wie bereits CARLGREN (1940) feststellte, haben die b-Rhabdoiden stets einen sehr langen (mehrfache Kapsellänge), bewaffneten Faden, der stets bei der Entladung ausgestülpt wird. Die p-Rhabdoiden haben dagegen einen unbewaffneten Faden, der meistens bei der Explosion nicht ausgestülpt wird. Da der Faden fest mit dem distalen Ende des Schaftes verbunden ist, bleibt er nicht wie bei den Aktinien (Scrmor 1969) in der Kapsel, sondern stets im Schaftende liegen. Sowohl bei den b- als auch bei den p-Rhabdoiden kann der Schaft einheitlich bewaffnet oder in Falt- und Hauptstïck gegliedert sein (vgl. Schmidt 1969). Während sich bei den b-Rhabdoiden das Faltstück lediglich durch kleinere Dornen von dem Hauptstück unterscheidet (Abb. 4), ist das Faltstück der p-Rhabdoiden im Gegensatz zu Curress (1955) nach meinen (elektronenmikroskopischen) Beobachtungen stets unbewaffnet. In der Schaftbewaffnung unterscheiden sich b- und p- Rhabdoiden nicht. Die Dornen sind in deutlichen Windungen angeordnet und kaum länger als der Schaft breit. Nach der Entladung wenden sich ihre Spitzen stets zur Kapsel hin (Abb. 2f, 4, 5). Bei elektronenoptischer Betrachtung läßt sich feststellen, daß fast alle Rhabdoiden ein kleines Faltstück haben (Abb. 3c). Bei lichtmikroskopischer Betrachtung ist aber nach Ausbildung und Länge des Faltstükkes folgende Gliederung möglich:

(1) b-Rhabdoiden: Der Schaft geht am distalen Ende konisch in den Endfaden über. Der lange hoplotele Endfaden wird stets ausgestïlpt.

(I) Ohne deutliches Faltstiuck: Der Schaft ist in nicht entladenem Zustand unterschiedlich lang, jedoch nicht länger als die Kapsel. Er geht am distalen Ende konisch in den langen Endfaden über (Abb. 2g), der vorwiegend in der unteren Kapselhälfte aufgerollt ist. In explodiertem Zustand ist der Schaft selten länger als eineinhalbmal so lang wie die Kapsel (Abb. 2f) oder gleichlang (Abb. 3b) oder kürzer. Dem konischen Ubergang zwischen Schaft und Faden in der geschlossenen Kapsel entspricht in explodiertem Zustand ein Ubergangsstück, das sich allmählich zu dem kleineren Querschnitt des Fadens verjüngt, aber noch mit kurzen typischen Schaftdornen besetzt ist ( $\mathrm{Abb}, 3 \mathrm{e}$ ). Der Faden ist mit kleinen unregelmäßig T-förmigen Dornen besetzt (Abb. 3d).

Abb. 3: a p-Rhabdoide, Gerardia savaglia, Mesenterialfilament (18000:1); $b$ kleine b-Rhabdoide I, Ceriantbus membranaceus, Mesenterialflament (mittlerer Abschnitt) (3130:1);c kleine b-Rhabdoide I, Ceriantbus membranacews, Mesenterialflament (mittlerer Abschnitt) (13 750:1); d große b-Rhabdoide $\mathrm{I}$, Cerianthus membranacevs, Mesenterialfilament (unterer Abschnitt) : Faden (17750:1); e große b-Rhabdoide 1, Cerianthus membranaceus, Mesenterialfilament (unterer Abschnitt : Schaftende und Faden (8700:1) 
(II) Mit deutlichem Faltstück: Der Schaft ist deutlich in Haupt- und Faltstïck gegliedert. Er ist häufig, auch in nicht explodiertem Zustand, länger als die Kapsel. Das proximale Faltstück hat feinere Schraubenwindungen und ist in einer halben oder ganzen Windung etwa im oberen Viertel der Kapsel aufgerollt (Abb. 4; vgl. CarLGREN
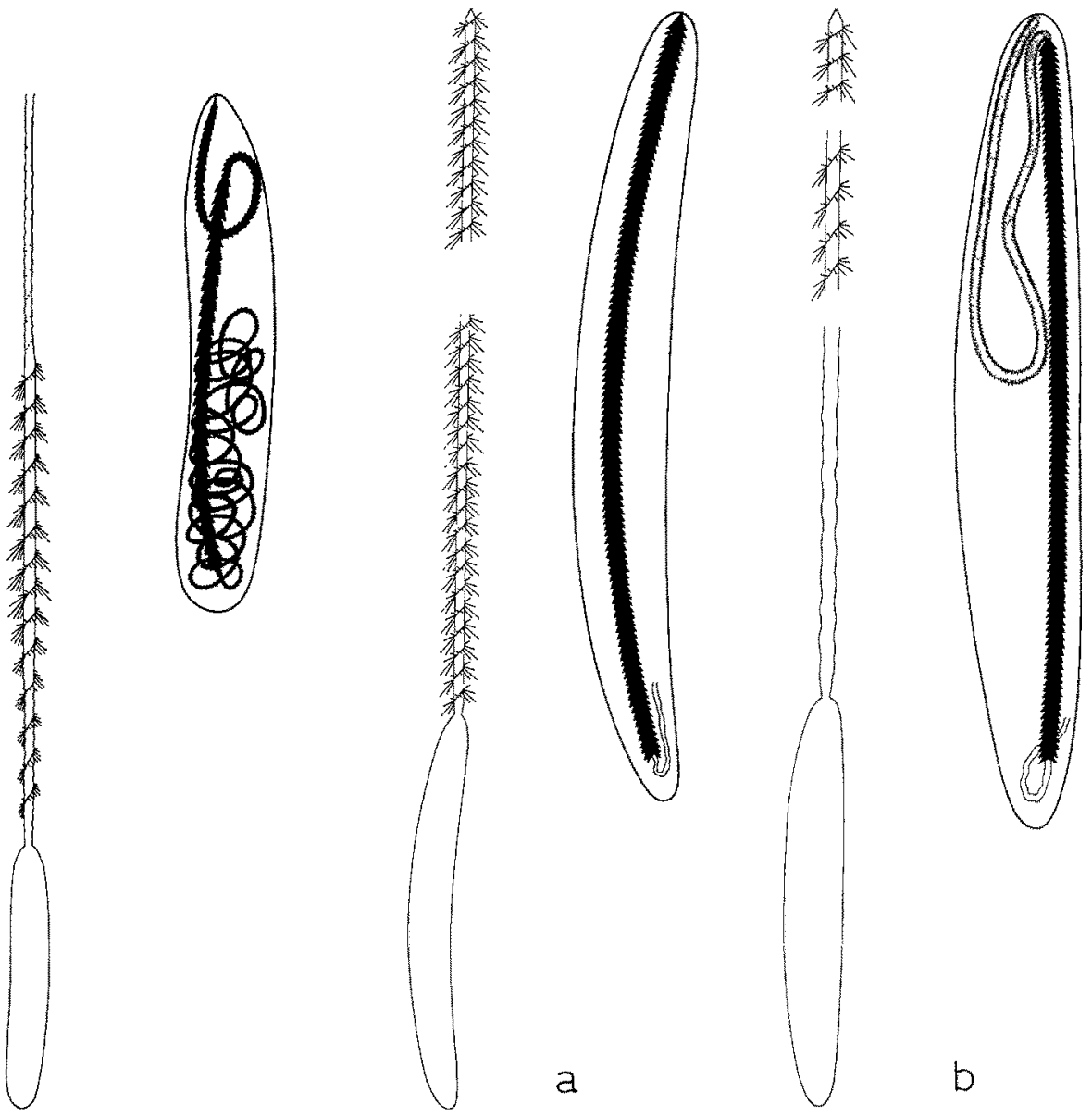

Abb. 4: b-Rhabdoide II, Pachycerianthus multiplicatus, Marginaltentakel $(800: 1)$

Abb. 5: a p-Rhabdoide I, Cerianthoides spec., Marginaltentakel $(850: 1)$, Faden im Schaft nicht eingezeichnet; $b$ p-Rhabdoide IIb, Cerianthoides spec., Labialtentakel (850:1), Faden im Schaft nicht eingezeichnet

1940, Fig. II 1, 2). In explodiertem Zustand erreicht der Schaft selten mehr als die doppelte Kapsellänge. Haupt- und Faltstück sind deutlich an den unterschiedlich langen Dornen zu erkennen (Abb. 4). Das Faltstïck nimmt etwa ein Drittel der gesamten Schaftlänge ein, selten weniger.

(2) $\mathrm{p}-\mathrm{R}$ h a bdoiden: Der unentladene Schaft ist am distalen Ende trichterförmig geöffnet. Der kurze anoplotele Endfaden verbleibt nach der Entladung im distalen Schaftende. 
(I) Ohne deutliches Faltstück: Der Schaft ist in nicht entladenem Zustand gerade gestreckt oder leicht gebogen und nimmt fast die ganze Länge der Kapsel ein (Abb. 5a). Der Schaft geht am distalen trichterartig geöffneten Ende abrupt in einen kurzen Faden über. In explodiertem Zustand kann der Schaft die zwei- bis vierfache Kapsellänge erreichen und ist gleichmäßig bewaffnet (Abb. 5a). Der kleinen trichterförmigen Öfnung des nicht ausgestülpten Schaftes entspricht in entladenem Zustand eine kurze konische Schaftspitze.

(II) Mit, deutlichem Faltstück: Das Faltstück hebt sich in nicht entladenem Zustand durch seine sehr dichten Schraubenwindungen deutlich von dem Hauptstück ab (Abb. 5b). Der Schaft reicht in der geschlossenen Kapsel stets bis zum Kapselende. Das Faltstïck ist je nach Länge leicht gebogen oder in der oberen Kapselhälfte in einer Windung aufgerollt (Abb. 2h, 5b). In explodiertem Zustand erreicht der Schaft je nach Länge des Faltstückes die 2- bis 16 fache Kapsellänge. Dabei nimmt das stets unbewaffnete Faltstück ein Zehntel bis drei Viertel der gesamten Schaftlänge ein. Nach den Längenverhältnissen von Haupt- und Faltstück kann unterschieden werden: einerseits Faltstück kürzer als Hauptstück, andererseits Faltstück länger als Hauptstück.

Dem Cnidom der Ceriantharien kommt eine ähnlich große diagnostische Bedeutung zu wie dem der Aktinien. Die wenigen hier aufgeführten Arten lassen sich alle an ihren Nesselkapseln unterscheiden. Bei der Revision der Ceriantharien (in Vorbereitung) sind zwei große Gruppen zu berücksichtigen. Eine Gruppe hat nur b-Rhabdoiden: Cerianthus mebranaceus, Cerianthus mana, Pacbyceriantbus multiplicatus. Zur zweiten Gruppe, die sich auch morphologisch unterscheidet, gehört Ceranthoides nov. gen. nov. spec., der neben b-Rhabdoiden vorwiegend $\mathrm{p}$-Rhabdoiden hat. Die neue Art und Gattung werden in Kürze ausführlich beschrieben. Innerhalb der ersten Gruppe ist vor allem Pachyceriantbus multiplicatus leicht an den b-Rhabdoiden II zu erkennen (vgl. CARLGREN 1940, Fig. II 1, 2), die im Gegensatz zu den p-Rhabdoiden II von Cerianthoides nicht in den Labial-, sondern in den Marginaltentakeln vorkommen. Wie bei den Aktinien sind die unterschiedlichen Nesselkapseltypen offenbar auch bei den Ceriantharien vorwiegend Merkmale der Gattungen und anderer höherer taxonomischer Einheiten. Cerianthus mawa und Cerianthus membranaceus unterscheiden sich nicht in den Nesselkapseltypen, sondern lediglich in den Größen ihrer Nesselkapseln.

\section{Corallimorpharia und Madreporaria}

Die Nesselkapseln der Madreporaria wurden bisher kaum als taxonomisches Merkmal gewertet. Das Cnidom besteht nach Weill (1934) und CARLGRen (1940) aus Spirocysten, holotrichen Haplonemen und b- und p-Mastigophoren (= Rhabdoiden). Nach den vorliegenden Befunden stimmen die Nesselkapseln der Madreporaria mit denjenigen der Corallimorpharia in Bau und Vorkommen vollkommen überein (vgl. RoBson 1953). Eine separate Beschreibung erübrigt sich daher.

\section{Die Háplonemen}

Die Haplonemen der Madreporaria und Corallimorpharia sind ausschließlich holotrich. Die atrichen Haplonemen, die WeILL (1934) für Corynactis viridis angibt, haben 

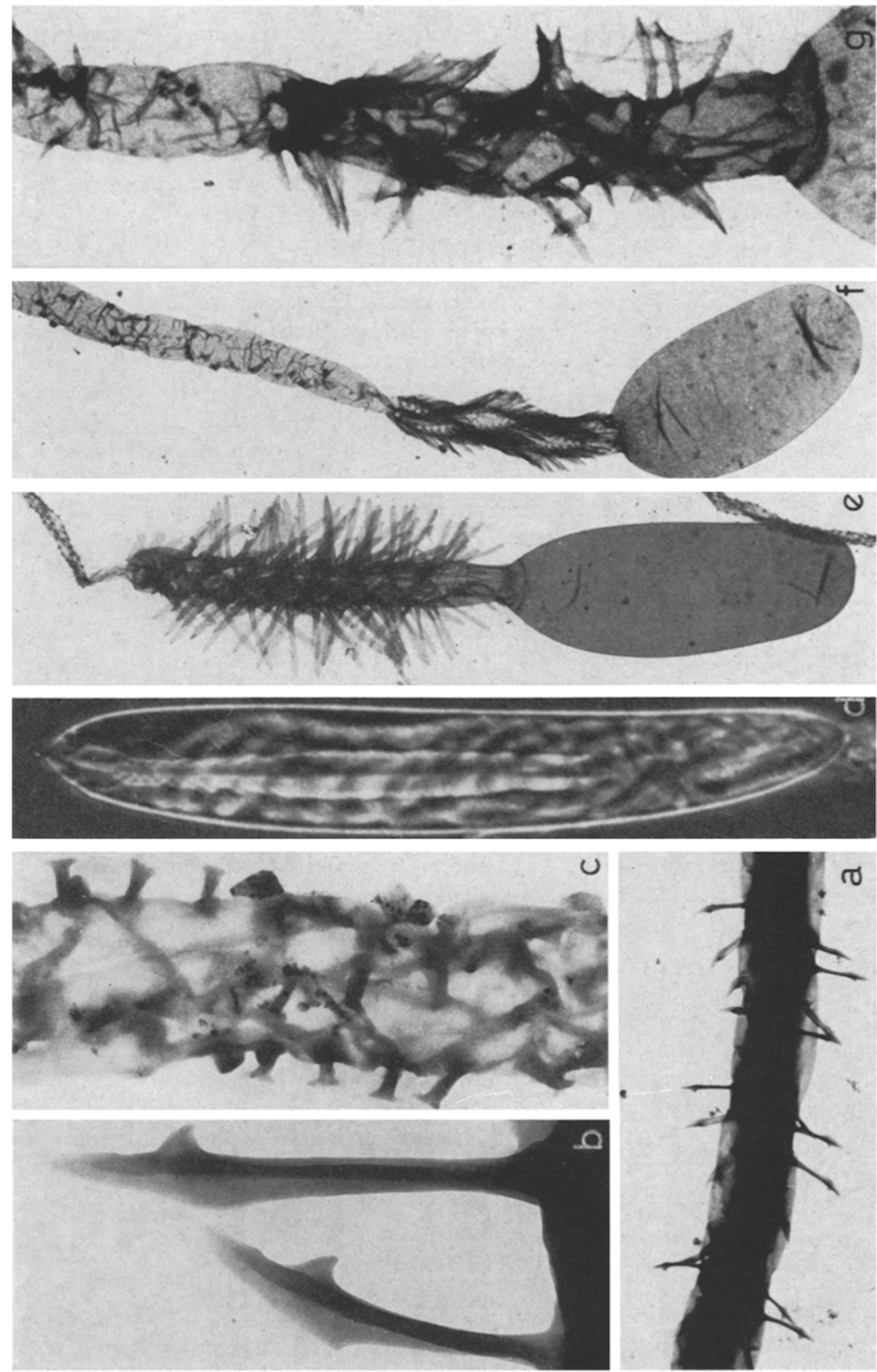
sich als holotriche Haplonemen erwiesen. Die nur bei den Corallimorpharia vorkommenden Haplonemen werden als holotriche Haplonemen II von den gemeinsamen großen holotrichen Haplonemen I unterschieden.

Holotriche Haplonemen I kommen zahlreich in den Akrosphären der Tentakeln, im Pharynx und in den Mesenterialfilamenten vor. In der nicht entladenen Kapsel ist der dicke kontrastreiche Schlauch deutlich zu erkennen und in unregelmäßigen Windungen angeordnet. Holotriche Haplonemen I zeichnen sich durch besonders differenzierte Dornen aus. Die Dornen tragen auf der proximalen Seite am distalen Ende je einen größeren und einen mehr distal angeordneten kleineren Sekundärdorn (Abb. 6a, b; 9a, b). Außerdem haben die Primärdornen etwa in Höhe des größeren Sekundärdorns seitlich zwei kleine Spitzen (Abb. 6b), die bei den Corallimorpharia allerdings nur angedeutet sind (Abb. 9a). Derart gegliederte Dornen waren unter den Anthozoen bisher nur von den Corallimorpharia bekannt (Corynactis viridis: vgl. Robson 1953, SKaER \& PrCKEN 1965). Der einzige Unterschied $z$ wischen Corallimorpharia und Madreporaria besteht darin, daß die Dornen der Madreporaria graziler sind und daher markantere Spitzen haben. Der Schlauch der Haplonemen I ist am äußersten distalen Ende leicht verjüngt und unbewaffnet. Cutress (1955) betrachtet diese Nesselkapsel daher als Heteroneme. Da der unbewaffnete Schlauchteil aber nur $1 / 250$ bis $1 / 300$ der Gesamtschlauchlänge ausmacht, wird die Nesselkapsel vorläufig weiter als Haploneme bezeichnet. Holotriche Haplonemen II kommen in den Mesenterialfilamenten, in den Randtentakeln und im Scapus verschiedener Corallimorpharia vor. Sie unterscheiden sich von den holotrichen Haplonemen I deutlich in Größe und Form und durch den wenig kontrastreichen, die ganze Kapsel in engen Windungen ausfüllenden Schlauch. Der geringe Phasenkontrast des Schlauches wird von den deutlich kleineren Dornen hervorgerufen, an denen Haploneme II leicht von Haploneme I zu unterscheiden ist.

\section{Die Heteronemen}

Die Heteronemen der Madreporaria und der Corallimorpharia sind völlig identisch. Sie bestehen aus b- und p-Rhabdoiden, die sich sehr markant unterscheiden.

(1) Die b-Rhabdoiden (Abb. 6f, g) kommen vorwiegend in den Tentakeln, im Scapus und im Pharynx vor. Der ausgestülpte Schaft ist kaum länger als die Kapsel, häufig wesentlich kürzer. Der Dornenbesatz ist weniger dicht als bei den p-Rhabdoiden, da die wenigen Dornenwindungen sehr steil ansteigen (vgl. Abb. 6e, f). Es bestehen so gut wie keine Unterschiede zwischen den Breiten von Schaft und Faden. Der breite Faden ist mit wenigen kleineren spitzen Dornen besetzt.

(2) Die p-Rhabdoiden (Abb. 6c, d, e) kommen sowohl in den Tentakeln, im Scapus und Pharynx, als auch in den Mesenterialfilamenten vor. Der auffallend breite

Abb. 6: a holotriche Haploneme 1, Coenocyathus dobrni, Mesenterialflament : im Innern noch nicht evaginierter Schlauch $(8380: 1) ; b$ holotriche Haploneme I, Coenocyatbus dobmi, Mesenterialfilament: Dornen $(43500: 1) ; c$ p-Rhabdoide, Astroides calycularis, Scapus : Faden $(25810: 1) ; d$ p-Rhabdoide, Coenocyathus dobrni, Tentakeln (2290:1); $\varepsilon$ p-Rhabdoide, Balanopbyllia verncaria, Scapus (2940:1); f b-Rhabdoide, Coenocyatbus dobrni, Scapus (2630:1); $g$ b-Rhabdoide, Caryopbyllia smithi, Scapus (12050:1) 

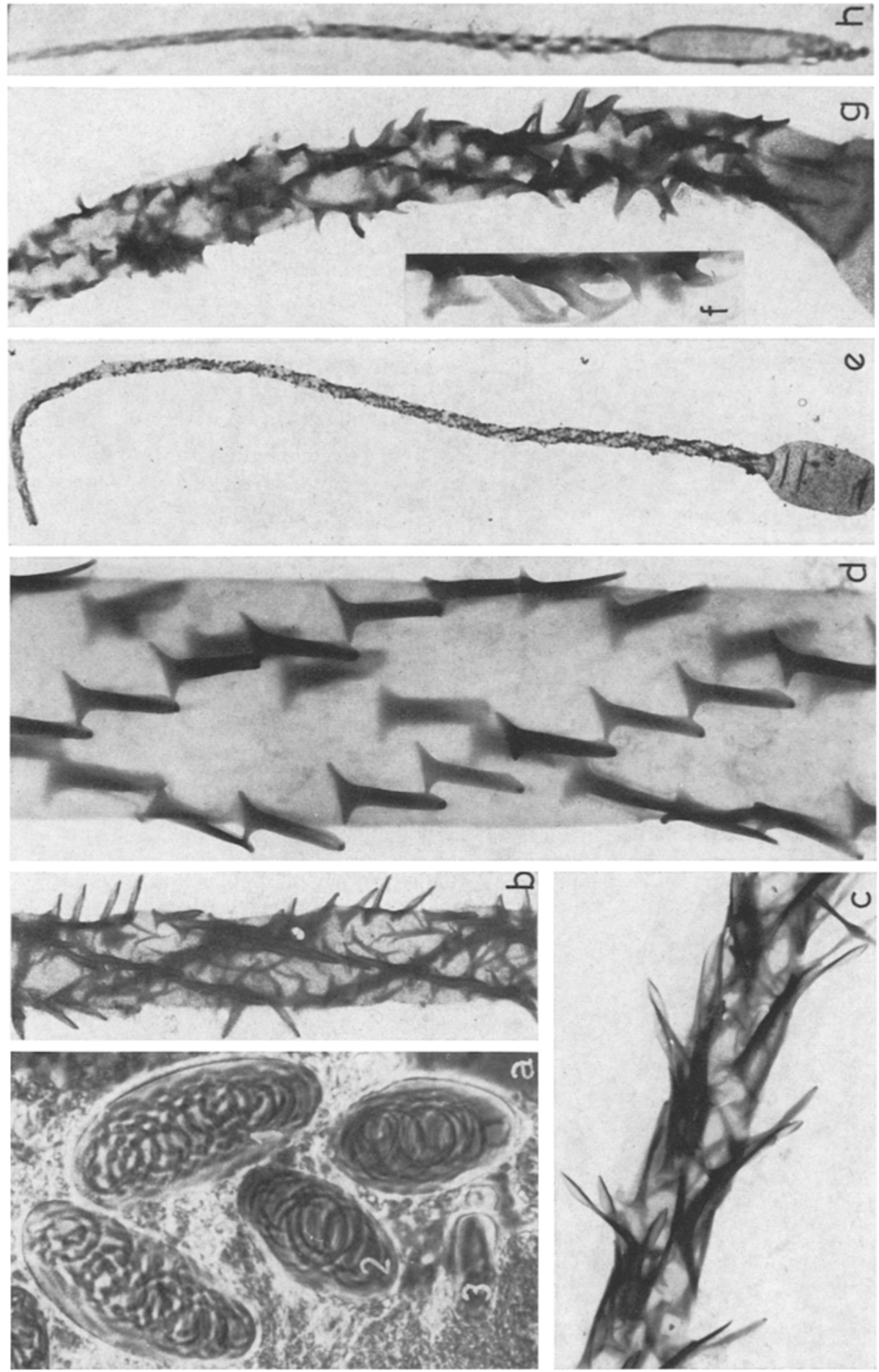
Schaft hat in der geschlossenen Kapsel eine deutliche trichterförmige Offnung, der ein sehr langer, meist die ganze Kapsel ausfüllender Faden entspringt. Der Schaft ist in explodiertem Zustand selten länger als die Kapsel, häufig gleichlang oder bedeutend kürzer. Er ist mit dichten, aber unterscheidbaren Dornenwindungen besetzt. Die Dornen stehen senkrecht auf der Schaftoberfläche. Am distalen Ende verjüngt sich der Schaft abrupt zu einem hoplotelen Faden, der dicht mit T-förmigen Dornen besetzt ist (Abb. 6c; vgl. Ronson 1953). Die Form der Dornen ist bei p-Rhabdoiden verschiedener Herkunft ziemlich unterschiedlich. Die T-Form kann regelmäßig oder unregelmäßig sein.

Weitere Unterteilungen der Rhabdoiden sind nicht möglich, da die morphologischen Voraussetzungen fehlen. Bei den großen p-Rhabdoiden der Mesenterialfilamente und der Akrosphären der Tentakeln ist nur die erste, am ausgestülpten proximalen Schaftende sehr steil ansteigende Windung mit anfangs kürzeren, aber immer länger werdenden Dornen versehen. Dieses Anfangsstück ist auch in der geschlossenen Kapsel zu erkennen (Abb. 6d). Es ist aber nicht mit dem Faltstück zu vergleichen, wie es bei einigen p-Rhabdoiden der Aktinien (SCHMIDт 1969) und Ceriantharien vorkommt, da das Anfangsstuick nicht gefaltet ist und nicht zur Verlängerung des Schaftes beiträgt. Bei einigen Rhabdoiden ist der Schaft so kurz, daß Schaft und Faden im Lichtmikroskop schwer zu unterscheiden sind. Da aber die proximale längere Bewaffnung deutlich abgesetzt ist, und sich die Dornen von Schaft (Dornen spitz) und Faden (Dornen am Ende T-förmig) auch morphologisch unterscheiden, handelt es sich definitionsgemäß (SCHMrDT 1969) um rhabdoide Heteronemen, die aber in geschlossenem Zustand leicht mit Haplonemen verwechselt werden können.

Die diagnostische Bedeutung des sehr einheitlichen Cnidoms der Madreporarien und Corallimorpharien liegt weniger in unterschiedlichen Nesselkapseltypen. Dafür unterscheiden sich aber Größen und Formen und zum Teil das Vorkommen der einzelnen Typen bei verschiedenen Familien, Gattungen und zum Teil Arten so beträchtlich, daß sie als diagnostisches Merkmal, im Gegensatz zur bisherigen Praxis, kaum außer acht $\mathrm{zu}$ lassen sind. Beispielsweise sind in einem bestimmten proximalen Abschnitt der Mesenterialfilamente der Caryophylliidae besonders große Haplonemen I und p-Rhabdoiden lokalisiert, während der distale, meist zooxanthellenhaltige Abschnitt über kleinere Nesselkapseln und weniger $\mathrm{p}$-Rhabdoiden verfügt. Bei den Dendrophylliidae sind die Nesselkapseln der Mesenterialfilamente schlanker und kleiner als bei den Caryophylliidae und größenmäßig einheitlich verteilt. Das Vorkommen von Haplonemen I in den Tentakeln ist ebenfalls bei verschiedenen taxonomischen Einheiten unterschiedlich. Die unterschiedliche Verteilung von Haplonemen ist ebenfalls ein wertvolles diagnostisches Merkmal der häufig sehr ähnlichen Corallimorpharia.

Abb. 7: a Große holotriche Haplonemen $\mathrm{Ia}$ (1) und $\mathrm{Ib}$ (2) und b-Rhabdoide (3), Parazoantbus axinellae, Scapus (1680:1); $b$ große holotriche Haploneme I, Gerardia savaglia, Scapus : Schlauchende $(8650: 1) ; c$ große holotriche Haploneme Ia, Parazoantbus axinellae, Scapus (9740:1); d große holotriche Haploneme Ib, Parazoantbus axinellae, Scapus (17000:1); $e$ kleine holotriche Haploneme, Gerardia savaglia, Scapus $(3380: 1)$; f kleine holotriche Haploneme, Parazoantbus axinellae, Scapus : Dornen $(42900: 1) ; g$ kleine holotriche Haploneme, Gerardia savaglia, Scapus : proximaler Schlauchteil (15290:1); $b$ b-Rhabdoide, Parazoanthus axinellae, Tentakel $(1595: 1)$ 

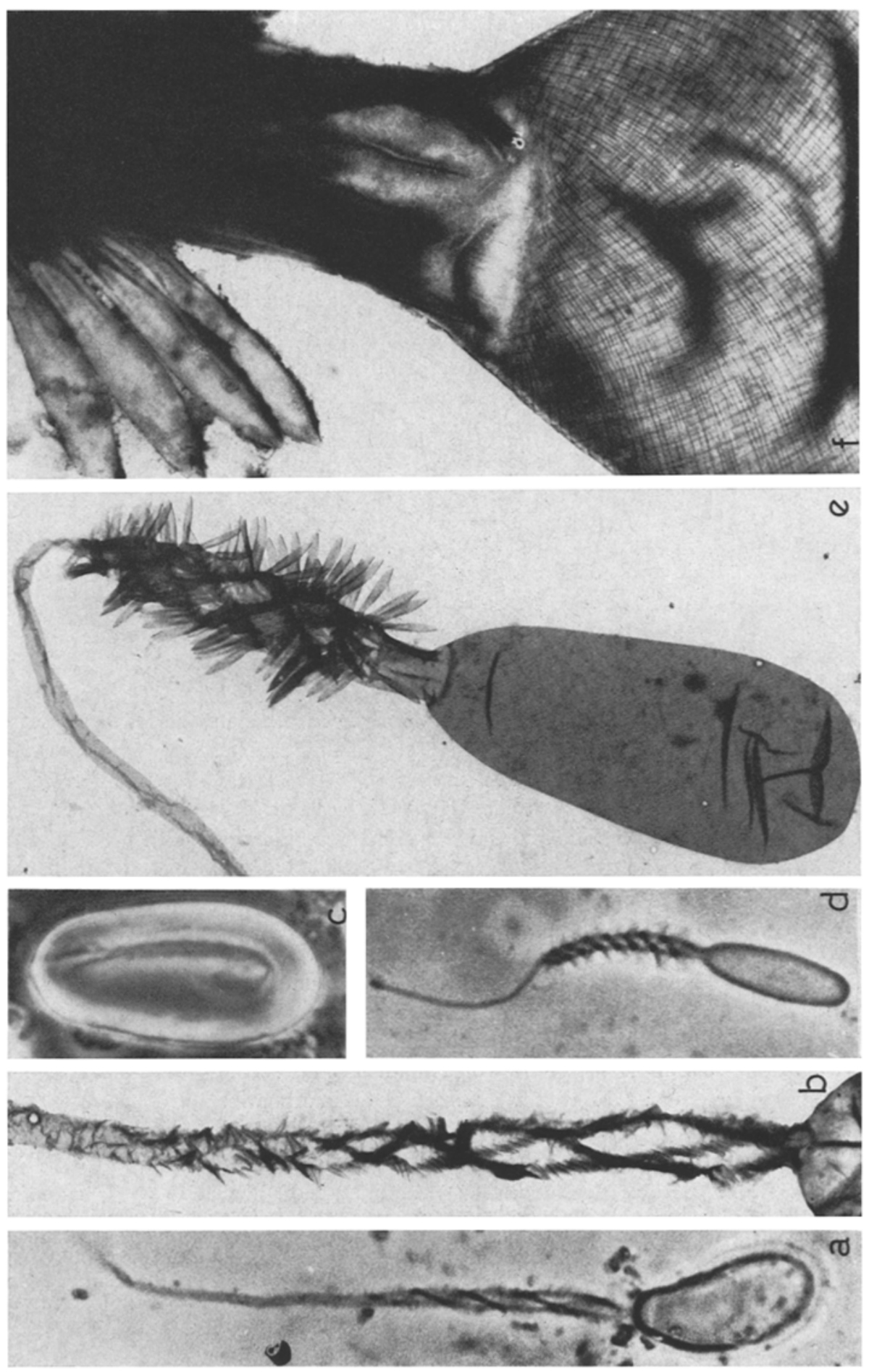


\section{Actiniaria}

Die Aktinien fallen durch eine bei anderen Anthozoenordnungen nicht zu erkennende Typen- und Formenvielfalt ihrer Nesselkapseln auf. Das Cnidom setzt sich aus Spirocysten, verschiedenen Haplonemen und sehr unterschiedlichen rhabdoiden Heteronemen zusammen. Letztere insbesondere waren bereits Gegenstand einer ausfuhrlichen Untersuchung (SCHMIDT 1969). Daher sollen hier nur ergänzend einige neue Ergebnisse mitgeteilt werden.

\section{Die Haplonemen}

(1) Atriche Haplonemen: Von allen bisher als "atrich" bezeichneten Haplonemen (WeILI 1934, HAND 1961) erwies sich lediglich jene große, dünnwandige, mit einem auffallend dicken, langen Schlauch versehene isorhize Haploneme aus dem Scapus von Sagartiogeton undatus und anderen Aktinien (Schmrd 1969, Abb. 1a-g) als wirklich atrich. Meine früheren Untersuchungen hatten diesen Befund nicht eindeutig erbracht. Diese auffallende Nesselkapsel kommt außer in Sagartiogeton undatus, sporadisch in den Akontien von Aiptasia diaphana und Sagartia troglodytes und im Scapus von Cereus pedunculatus, Halcampa chrysantbellum, Bolocera tuediae und Anthopleura rubripunctata vor.

(2) Holotriche $\mathrm{Haplo}$ emen: Am Beispiel von Cereus pedunculatus konnte festgestellt werden, daß die beiden früher als atriche und holotriche, inzwischen aber von WESTFALL (1965, Abb. 7, 8) als eindeutig holotrich erkannten Haplonemen aus den Fangtentakeln sich geringgradig in der Dichte der Dornenwindungen und der Länge der Dornen unterscheiden. Beide Nesselkapseln haben einfach-spitz auslaufende Dornen. Die isorhizen Haplonemen der Akrorhagen so unterschiedlicher Arten wie Actinia equina, Anemonia sulcata und Antbopleura ballii haben gleichmäßig T-förmig ausgebildete Dornen. Die Dornen der anisorhizen Haplonemen aus dem Scapus von Anthopleara rubripunctata sind dagegen ungewöhnlich groß und am distalen Ende einfach spitz auslaufend.

\section{Die Heteronemen}

Die sogenannten atrichen Haplonemen aus den Tentakeln von Protanthea simplex erwiesen sich als eindeutige b-Rhabdoiden. Sie verfügen über einen auch in der nicht explodierten Kapsel im Phasenkontrast zu erkennenden sehr kurzen Schaft. Die Schaftlänge beträgt in der Kapsel etwa ein Zehntel und in explodiertem Zustand etwa ein Achtel der Kapsellänge. Der Heteronemencharakter kommt in der deutlich abgesetzten

Abb. 8: a Spezielle b-Rhabdoide, Gerardia savaglia, Scapus (1280: 1); $b$ spezielle b-Rhabdoide, Gerardia savaglia, Scapus (4090:1); c spezielle b-Rhabdoide, Gerardia savaglia, Scapus $(1920: 1)$; $d$ p-Rhabdoide, Parazoantbus axinellae, Mesenterialfilament $(1240: 1)$; e p-Rhabdoide, Gerardia savaglia, Mesenterialflament (6100:1);f p-Rhabdoide, Parazoanthus axinellae, Mesenterialfilament $(20400: 1)$ 
längeren Schaftbewaffnung und den im Gegensatz zu den spitzen Schaftdornen Tförmigen Fadendornen zum Ausdruck.

In der gleichen Weise unterscheiden sich die besonderen b-Rhabdoiden der Akontien der Aiptasiidae (Schmid 1969, Abb. 2c, Schmmt 1972b, Abb. 7c, d), deren Fadendornen T-förmig sind, von den übrigen b-Rhabdoiden mit spitzen Fadendornen.

Bei einer Phymanthusart aus dem Indischen Ozean wurden im Mauerblatt und in den Mesenterialfilamenten neben normalen p-Rhabdoiden A Nesselkapseln gefunden, die alle typischen Merkmale der p-Rhabdoiden A (SCHMIDT 1969) haben, jedoch über ein deutliches Faltstück verfügen. Die Nesselkapsel wird daher p-Rhabdoide A2 bezeichnet. Sie wird in einem anderen Zusammenhang ausfubrlich beschrieben und dargestellt.

Bei einer noch nicht näher beschriebenen Aktinie, die einer Edwardsiide äußerlich sehr ähnlich ist, konnten keinerlei p-Rhabdoiden beobachtet werden, sondern nur hoplotele b-Rhabdoiden. In den Mesenterialfilamenten kommen zwei in Kapselform, in der Dichte der Dornenwindungen und in der Länge der Dornen voneinander abweichende b-Rhabdoiden vor. In den Mesenterialfilamenten von Edwardsia-Arten kommen dagegen kleine und große $b-R h a b d o i d e n$ und $\mathrm{p}-$ Rhabdoiden $\mathrm{A}$ vor. Dieses Cnidom kennzeichnet die abgeleiteten Actiniaria (vgl. Diskussion), die Endomyaria, zu denen die echten Edwardsien dem Nesselkapselbefund zufolge gehören.

Die übrigen Befunde haben keine größere diagnostische Bedeutung. Die genaue Verbreitung der p-Rhabdoiden A2 ist noch zu untersuchen. Der unterschiedliche Dornenbefund bei den anisorhizen Haplonemen von Anthopleura rubripunctata und den typischen Akrorhagenhaplonemen anderer Aktinien könnte Zweifel darüber aufkommen lassen, ob die taxonomische Stellung von Anthopleura rubripunctata gerechtfertigt ist (vgl. ScHMIDT 1972b).

\section{Zoantharia}

Die Nesselkapseln der Zoantharia wurden von SEIFerT (1928) und WeILl (1934) unterschiedlich klassifiziert. Schon bald stellte sich heraus, daß SEIFERTs Klassifizierung nicht eindeutig und daher für taxonomische Zwecke unbrauchbar war (vgl. WeILL 1934, Tischbierek 1936, Carlgren 1937). Nach Weill (1934) besteht das Cnidom der Zoantharia aus Spirocysten, holotrichen Haplonemen und rhabdoiden Heteronemen (vgl. CarLGRen 1938).

\section{Die Haplonemen}

Die Zoantharien verfügen über verschiedene, ausschließlich holotriche Haplonemen, die als I und II unterschieden werden. In Scapus, Tentakeln, Pharynx und Mesenterialfilamenten kommen große holotriche Haplonemen I vor. Die Größe und Form der Kapseln sowie die Länge und Form der Dornen (Abb. 7a-d) können bei den verschiedenen Arten unterschiedlich ausfallen. Die größere Haploneme Ia zeigt, bedingt durch die längere Bewaffnung, in nicht explodiertem Zustand einen deutlich dickeren Schlauch und ist daher leicht von der etwas kleineren Haploneme Ib zu unterscheiden (vgl, Abb. 7a). Die Form der Dornen ist ebenfalls verschieden. Haploneme la hat 
lange, an der distalen Hälfte löffelartig verbreiterte zugespitzte Dornen (Abb. 7c). Die Dornen von Haploneme Ib sind wesentlich kürzer und distal nicht verbreitert ( $\mathrm{Abb} 7 \mathrm{~d}$ ). Bei einigen Arten kommen Ubergangsstadien vor, die einfach als Haplonemen I bezeichnet werden (Abb. $7 \mathrm{~b}$ ).

Außer diesen großen Haplonemen kommen in Scapus und Mesenterialfilamenten bisher nicht beschriebene, wesentlich kleinere Haplonemen II mit relativ kurzem Schlauch vor (Abb. 7e). Die Dornen laufen bei einigen Arten am distalen Ende T-förmig aus. Bei anderen Arten sind sie spitz und leicht gebogen (Abb. 7f, g). Sie nehmen stets deutlich gegen das distale Schlauchende an Größe ab.

\section{Die Heteronemen}

Die thabdoiden Heteronemen der Zoantharia können ebenfalls als b- und pRhabdoiden unterschieden werden:

(1) Die $b-R h a b$ doiden kommen in sehr verschiedenen Größen und Formen vor und sind stets hoplotel. Der Schaft ist etwa kapsellang und relativ breit. Die Schattornen sind spitz. Bei den meisten von WEILI (1934, Abb. 428; 432 B, C; 430) abgebildeten "mikrobasischen Mastigophoren" handelt es sich um b-Rhabdoiden, die aber im Gegensatz zu WeILL (1934) und Carlgren (1940) hoplotele Fäden haben (Abb. 7h).

Tief im Ektoderm des Scapus kommt eine spezielle b-Rhabdoide vor (Abb. 8a-c), die im Gegensatz zu Serferts Abbildung (1928, Abb. 8) nur einen sehr kurzen spärlich bewaffneten Faden hat. Der Schaft ist deutlich anisodiametrisch, und die Dornen sind an dem schmaleren Schaftende etwas kürzer. Daher ist der Schaft bei einigen Arten, z. B. Parazoantbus axinellae, nicht so deutlich abgesetzt und könnte als Schlauch einer anisorhizen Haploneme aufgefaßt werden. Bei Gerardia savaglia und Epizoanthus conchi sind aber Schaft und Faden auf Grund ihrer Bewaffnung und Breite eindeutig zu unterscheiden, weshalb die Nesselkapsel im Gegensatz zu SEIFERT (1928) als Heteroneme einzustufen ist. WEILL (1934) hat diese Nesselkapsel offenbar nicht beobachtet. Tentakeln, Pharynx und Scapus verfügen über gewöhnliche b-Rhabdoiden (Abb. $7 \mathrm{~h}$ ). In den Mesenterialfilamenten können besonders große b-Rhabdoiden neben Haplonemen und p-Rhabdoiden auftreten (vgl. SeIfeRt 1928, Abb. 12, Carlgren 1937, 1938).

(2) Di e p-R habdoiden der Zoantharia sind sehr einheitlich gestaltet und auch größenmäßig wenig variabel (Abb. $3 a, 8 d-f)$. Sie treten in den Filamenten, im Pharynx und im Scapus auf. Die Kapsel ist auffallend dünnwandig, und der Inhalt zeigt kaum Phasenkontrast (vgl. Schмid 1969, Abb. 7c). Daher sind Schaft und Faden in nicht explodiertem Zustand gut zu erkennen. Der Schaft reicht in nicht ausgestülptem Zustand etwa bis zur Mitte der Kapsel. Der explodierte Schaft ist etwa kapsellang, selten länger und häufiger kürzer. Die Dornen sind in deutlichen. Windungen angeordnet und stehen senkrecht zur Schaftoberfläche (Abb. 8d; vgl. SCHMD' 1969, Abb. 7b). WeILl (1934) und CARLgReN (1940) sind bei ihrer Beurteilung der Fadenbewaffnung wohl von diesen p-Rhabdoiden ausgegangen, denn sie sind anoplotel. Andere p-Rhabdoiden wurden bisher bei den Zoantharien nicht beobachtet.

Die diagnostische Bedeutung der Nesselkapseln der Zoantharia liegt vor allem in 

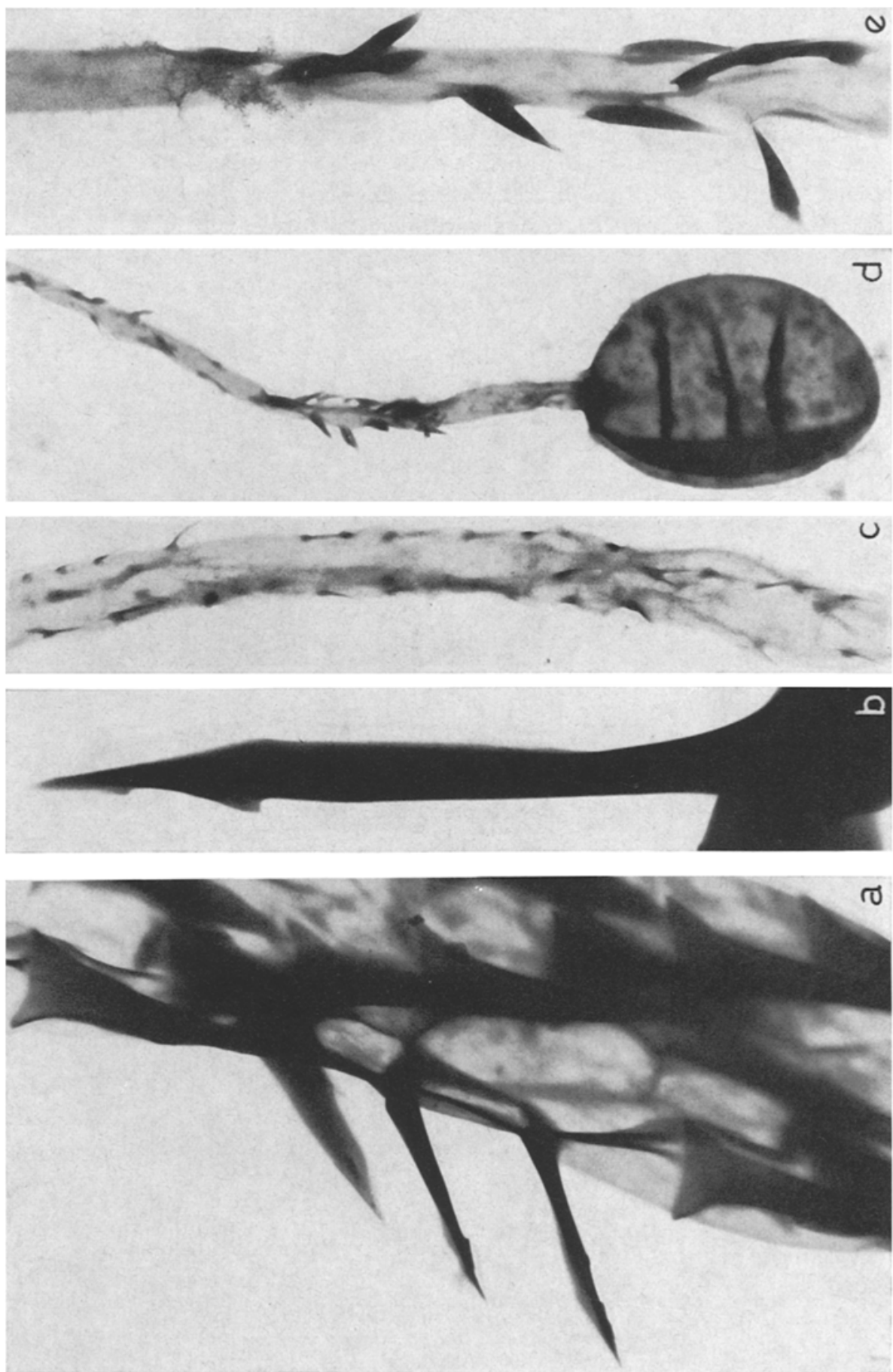
den verschiedenen Formen der b-Rhabdoiden und der Haplonemen sowie der Verteilung der p-Rhabdoiden. Bei einer dringend erforderlichen Revision werden zwei größere taxonomische Einheiten $z u$ unterscheiden sein. Nach meinen bisherigen Untersuchungen kommen bei Palythoa-Arten und eventuell auch bei anderen Zoanthidae (vgl. Carlgren 1937, 1938) typische große, schlanke b-Rhabdoiden in den Mesenterialfilamenten (vgl. SEIFERT 1928) und bei einigen Arten p-Rhabdoiden im Scapus vor. Bei einer anderen Gruppe (Epizoanthus, Parazoantbus, Gerardia) kommen in den Mesenterialfilamenten keine großen b-Rhabdoiden vor. p-Rhabdoiden treten bei dieser Gruppe nur in Pharynx und Mesenterialfilamenten auf. Die oben beschriebenen speziellen rundlichen b-Rhabdoiden, die nach meinen bisherigen Beobachtungen bei Palythoa fehlen, sind charakteristisch für diese Gruppe. Bei Parazoanthus treten im Gegensatz zu Gerardia und Epizoanthus im Scapus die beiden Formen a und b der großen Haploneme I nebeneinander auf (Abb. 7a).

\section{Antipatharia}

Das Cnidom der Antipatharia wurde von Dantan (1921), Trschbierek (1936) und Cartgren (1940) untersucht. Nach den Befunden dieser Autoren bestehen die Nesselkapseln der Antipatharien aus Spirocysten, holotrichen Haplonemen, "basitrichen “ Haplonemen und "mikrobasischen p-Mastigophoren" (= p-Rhabdoiden). Wie bei allen anderen Anthozoen handelt es sich bei den „basitrichen“ Haplonemen der Antipatharien ebenfalls stets um hoplotele b-Rhabdoiden. Holotriche Haplonemen kommen sehr selten vor. Sie fehlen in den hier untersuchten Arten und können daher in dieser Arbeit nicht beschrieben werden. DaNTAN (1921) fand holotriche Haplonemen im tieferen Gewebe des Ektoderms und des Pharynx von Leiopatbes glaberrima und TischBierex (1936) im Pharynx von Antipathes valdiviae.

\section{Die Heteronemen}

Die Untersuchungen haben ergeben, daß bei den Antipatharien drei verschiedene rhabdoide Heteronemen vorkommen.

(1) In den Mesenterialfilamenten und weniger häufig im Pharynx kommen kleine (5-7 $\mu \mathrm{m})$, rundliche Heteronemen vor (Abb. 9d). Der lange Schlauch ist leicht anisodiametrisch und auf Grund unterschiedlicher Bewaffnung in Schaft und Faden zu unterteilen. Der Schaft ist etwa so lang wie die Kapsel. Er ist proximal unbewaffnet und hat distal wenige, aber sehr markante spitze Dornen (Abb. 9e), die mit einer dreieckigen Basis an der Schaftoberfläche verankert sind. Der kaum dünnere Faden hat wesentlich

\footnotetext{
Abb. 9: a Holotriche Haploneme I, Rbodactis rbodostoma, Mesenterialfilament $(6600: 1)$; $b$ holotriche Haploneme I, Rbodactis rhodostoma, Mesenterialfilament : Dorn $(22320: 1)$; c p-Rhabdoide, Eucirripatbes anguina, Mesenterialfilament : hoploteler Faden (13725:1); $d$ spezielle rhabdoide Heteroneme, Euantipatbes dichotoma, Mesenterialfilament (8050:1); $e$ spezielle rhabdoide Heteroneme, Euantipatbes dichotoma, Mesenterialflament : Schaft
} $(16270: 1)$ 
feinere Dornen. Dornenwindungen sind weder am Schaft noch am Faden eindeutig zu erkennen. Die geschlossene Kapsel weist deutlichen Phasenkontrast auf. Der gesamte Schlauch ist wie bei den rhabdoiden Heteronemen der Octocorallia in zahlreidhen Windungen in der Kapsel aufgerollt. Schaft und Faden sind in der geschlossenen Kapsel kaum zu unterscheiden. Es ist daher nicht möglich zu entscheiden, ob es sich um b-oder um p-Rhabdoiden handelt.

(2) In allen Körperbereichen sind in unterschiedlicher Größe b-Rhabdoiden zu finden. Sie sind sämtlich hoplotel. Der Faden trägt kleine einfache spitze Dornen. Der Schaft erstreckt sich häufig fast über die ganze Länge der Kapsel, die von den engen Windungen des langen Fadens vollständig ausgefüllt wird. In explodiertem Zustand ist der Schaft etwa kapsellang. Die Schaftdornen unterscheiden sich nur wenig von denjenigen der $\mathrm{p}$-Rhabdoiden. In den Tentakeln und im Scapus kommen teilweise bRhabdoiden mit kürzeren Schäften vor. Im allgemeinen unterscheiden sich die b-Rhabdoiden der Antipatharia kaum von den normalen b-Rhabdoiden der Zoantharia. Die Antipatharia haben im Gegensatz zu den Zoantharia wie die Actiniaria kleine b-Rhabdoiden in den Mesenterialfilamenten.

(3) p-Rhabdoiden sind stets in den Mesenterialfilamenten und teilweise auch im Pharynx zu beobachten. Bei einigen Arten kommen sie im Gegensatz zu TrschirereK (1936) auch in den Tentakeln und im Pharynx vor. Sie stimmen mit Ausnahme des Fadens sehr genau mit den p-Rhabdoiden A der Actiniaria (Schmidt 1969, Abb. 7b, c, 8) und der Zoantharia überein. Die p-Rhabdoiden der Antipatharia haben ebenfalls kaum Phasenkontrast. Der kurze Schaft reicht in der geschlossenen Kapsel etwa bis zu deren Mitte. Der lange dünne Faden ist in zahlreichen Schrägwindungen aufgerollt. In explodiertem Zustand ist der Schaft selten länger als die Kapsel. Die Dornen stehen senkrecht zur Schaftsoberfläche und sind in deutlichen Windungen angeordnet. Der dünne Faden ist relativ lang und mit kleinen, schmalen, spitzen Dornen versehen, die bei keinen Rhabdoiden anderer Anthozoen bisher beobachtet wurden (Abb. 9c).

Der diagnostische Wert der beschriebenen Nesselkapseln besteht vor allem in dem unterschiedlichen Vorkommen der kleinen rhabdoiden Heteronemen und der unterschiedlichen Verteilung der" p-Rhabdoiden. Die kleinen rhabdoiden Heteronemen wurden bisher als offenbar artspezifisches Merkmal je einer Euantipathes- und einer Eucirripathes-Art beobachtet. Bei anderen Arten der gleichen Gattung fehlen sie. Ein wesentliches Merkmal ist das seltene Vorkommen von p-Rhabdoiden in den Tentakeln oder im Scapus. Die p-Rhabdoiden des Scapus sind häufig wesentlich größer als diejenigen der Mesenterialfilamente. Bei einigen Arten bestehen auch geringgradige Unterschiede in dem Verhältnis von Kapsel- und Schaftlänge.

Möglicherweise beobachtete CARLGREN (1940) die kleinen, rundlichen rhabdoiden Heteronemen in den Mesenterialfilamenten yon Cirripathes densiflora. CARLGREN vermutcte, daß es sich um atriche Haplonemen handelt. Da er die Nesselkapsel aber nur in einem einzigen Mazerationspräparat finden konnte, nahm er an, daß sie nicht von der Antipatharie stammt. Bei einigen Arten konnte ich außer den hier beschriebenen Nesselkapseln ebenfalls eine sehr kleine Nesselkapsel in den Mesenterialfilamenten beobachten, die den kleinen Haplonemen mancher Aktinien (Schмгd 1969, Abb. 1d) sehr ähnlich ist. Diese Nesselkapsel konnte zwar elektronenoptisch noch nicht untersucht werden, sie unterscheidet sich aber sehr deutlich in der Schattbewaffnung, in der 
Kapselform und in der Schlauchlänge von den hier beschriebenen kleinen rhabdoiden Heteronemen.

\section{DISKUSSION UND SCHLUSSFOLGERUNGEN}

\section{Vorbemerkungen}

Bei einer zukünftigen Neuordnung der Nesselkapseln ist die Bildung monophyletischer Kategorien anzustreben. Dabei ist der monophyletische Ursprung der in einer Kategorie vereinten Nesselkapseln anhand von abgeleiteten, sogenannten apomorphen Merkmalen der einzelnen Nesselkapseln zu begründen. Weisen mehrere Kategorien neben unterschiedlichen auch gemeinsame abgeleitete Merkmale ihrer Nesselkapseln auf, so sind diese synapomorph. Solche Kategorien können dann in einer höheren Kategorie zusammengefaßt werden. Ein nur bei einem einzigen Nesselkapseltyp vorkommendes abgeleitetes Merkmal ist autapomorph. Lediglich apomorphe Merkmale weisen auf einen monophyletischen Ursprung hin und sind somit konstitutiv. Ursprüngliche, sogenannte plesiomorphe Merkmale können zwar auf eine bestimmte Abstammung deuten, haben aber für die Bildung monophyletischer Gruppen keine Bedeutung. Nur das Fehlen von plesiomorphen Merkmalen kann unter Umständen apomorph sein. Auf verschiedenen taxonomischen Niveaus kann ein und dasselbe Merkmal entweder plesiomorph oder apomorph sein. Zum Beispiel können, wie unten gezeigt wird, innerhalb der Anthozoen Nesselkapseln mit T-förmigen Dornen symplesiomorph sein. Innerhalb der Anthozoen sind T-förmige Dornen daher kein konstitutives Merkmal. Sollten sie aber bei keinen anderen Cnidariern zu finden sein, so können sie innerhalb der Cnidarier als autopomorphes Merkmal der Anthozoen gewertet werden. Es ist weiterhin möglich, daß bestimmte Merkmale (Nesselkapseln) unterschiedliche Ausprägungsstufen erlangt haben oder zu verschiedenen Zeiten angelegt wurden. Solche Merkmale sind dann relativ apomorph in der abgeleiteten Gruppe aber plesiomorph. Zum Beispiel ist die Ausbildung großer b-Rhabdoiden in den Mesenterialfilamenten von Aktinien und Zoantharien ein relativ synapomorphes Merkmal der "jüngsten“ Aktinien und der "frühen “ Zoantharien (Tab. 1), das aber von den "späteren“ Zoantharien nicht mehr angelegt wurde. Innerhalb der Aktinien sind diese Nesselkapseln daher ein autapomorphes Merkmal der Endomyaria, da sie bei keinen anderen Aktinien vorkommen (vgl. Scrmor 1972b). Innerhalb der Zoantharien sind die großen bRhabdoiden der Mesenterialfilamente dagegen plesiomorph und ihr Fehlen bei den "späten" Zoantharien und bei den Antipatharien synapomorph.

Die vorliegenden Befunde zeigen, daß WeILls (1934) Nesselkapselkategorien keine konstitutiven Gruppen darstellen (vgl. Schмidt 1969). Nicht alle Nesselkapseln lassen sich in konstitutiven Kategorien vereinen. Für die phylogenetische Systematik sind nur die Nesselkapseltypen von Bedeutung, die, auf Grund von synapomorphen Merkmalen $z u$ einer konstitutiven Kategorie vereint, den monophyletischen Ursprung einer bestimmten Artengruppe anzeigen. Nur so ergibt sich die Möglichkeit, die Nesselkapseln als konstitutive Merkmale zu verwenden. Die zeitbedingte grobe Vereinheitlichung von sämtlichen Nesselkapseltypen, die WEILL (1934) in seinem allgemein gül- 
tigen System vornahm, ist daher für die phylogenetische Systematik unbrauchbar (vgl. WERNER 1965). Wie bei anderen Merkmalen ergeben erst die spezifischen Unterschiede oder Übereinstimmungen der einzelnen Nesselkapseltypen besonders der unteren Kategorien Einblicke in phylogenetische Verwandtschaftsbeziehungen.

\section{Die phylogenetische Morphogenie der Nesselkapseln und ihre Bedeutung als konstitutive Merkmale}

\section{Atriche Haplonemen}

Entgegen den Befunden verschiedener neuerer Arbeiten (Cutress 1955, PICKEN \& SKAER 1966, WeStPaLl 1965), wonach sich zahlreiche von WeIll (1934) als Atriche gekennzeichneten Haplonemen als holotrich erwiesen haben, hat sich bei den vorliegenden Untersuchungen ergeben, daß bei den Ceriantharia und bei den Actiniaria echte Atriche vorkommen. Sie stimmen in mehreren Merkmalen überein. Die Atrichen sowohl der Ceriantharia als auch der Actiniaria sind auffallend groß, haben duinnwandige Kapseln und etwa den gleichen Phasenkontrast. Sie können in allen Körperbereichen vorkommen, besonders häufig aber im Scapus.

Die Atrichen gelten allgemein als die "morphologisch und evolutiv einfachsten" Nesselkapseltypen (WERNER 1965). Aus ihnen sollen sich je nach morphologischem Differenzierungsgrad die anderen Nesselkapseltypen entwickelt haben (vgl. WeILL, 1934, Fig. 8b; CARLGREN 1940, p. 55). Es ist jedoch fraglich, ob die Evolution der Nesselkapseln stets höhere morphologische Differenzierung bedeutete (CARLGREN 1940, vgl. SCHMIDT \& BÉRESS 1971). Ob die Bewaffnung der Holotrichen oder die fehlende Bewaffnung der Atrichen apomorph ist, konnten daher auch WEILL (1934) und CARLGReN (1940) nicht entscheiden. Daß es sich bei den Atrichen um ein plesiomorphes Merkmal handelt, könnte aus der Funktion hervorgehen. Die Atrichen der Ceriantharien dienen in erster Linie der Herstellung der Wohnröhre, die im ersten Stadium nur aus Schleim und unzähligen entladenen und zu einem äußerst festen Gewebe verschlungenen Schläuchen von Atrichen besteht (Abb. 2b). Nach vollständiger Entfernung der alten Wohnröhre ermöglicht bei starker Reizung die massenhafte Explosion von Atrichen im Ablauf von Sekunden die Herstellung einer neuen Hülle. Die Actiniaria bilden keine mit der Wohnröhre der Ceriantharia vergleichbare Nesselkapselhülle. Das sporadische Vorkommen bei den Aktinien zeigt, daß die Atrichen bei den Actiniaria offenbar an Bedeutung verloren haben und innerhalb der Anthozoen bei Ceriantharia und Actiniaria symplesiomorph sind.

\section{Holotriche Haplonemen}

Holotriche Haplonemen wurden bei allen Ordnungen der Hexacorallia gefunden. Sie unterscheiden sich wie kein anderer Nesselkapseltyp in der Form der Dornen und in ihrem Vorkommen. Die Ceriantharia haben Holotriche im Scapus und nach CARLGREN 
(1940) selten auch in den Labialtentakeln und im Pharynx. Die Dornen sind klein, kompakt und deutlich T-förmig (Abb. 2d, e). Ahnliche Dornen haben die holotrichen Haplonemen der Akrorhagen der Aktinien. Die übrigen Holotrichen der Aktinien haben spitze Dornen. Sämtliche Holotriche wurden bei den Aktinien nur im Ektoderm gefunden. Bei den übrigen Anthozoen sind die Holotrichen die einzige Haplonemenform. Auch die Corallimorpharia haben entgegen früheren Auffassungen (WEILL 1934, Carlgren 1940) keine Atrichen. Außerlich den Atrichen ähnliche Holotriche II der Corallimorpharia sind nur an der proximalen Schlauchhälfte atrich, an der distalen Hälfte aber holotrich. Bei den Madreporaria, Zoantharia und Antipatharia können Holotriche mit Ausnahme der Mesenterialfilamente der Antipatharia (DANTAN 1921, TISCHBIEREK 1936) in allen Körperbereichen vorkommen. Die Dornenstrukturen sind bei den einzelnen Ordnungen zum Teil sehr spezifisch. Bei sämtlichen Holotrichen ist die Bewaffnung im Lichtmikroskop zu erkennen. Für die Beurteilung des atrichen $\mathrm{Zu}$ standes ist im Gegensatz zu WERNER (1965) die Beschaffenheit der Schlauchoberfläche kein Kriterium. Die wenigen atrichen Haplonemen der Anthozoen sind jedoch sehr einfach an der auffälligen Größe, dem Phasenkontrast und häufig an der Beschaffenheit des nicht ausgestülpten Schlauches von den Holotrichen zu unterscheiden (vgl. Abb. 2a). Von den mannigfaltigen Dornenstrukturen der holotrichen Haplonemen und der Heteronemen lassen sich nur dic T-förmigen Dornen vergleichen. Sie wurden als symplesiomorphes Merkmal bei verschiedenen Haplonemen und Heteronemen fast aller Anthozoen mit Ausnahme der Antipatharia gefunden. Der Übergang von T-förmigen zu spitzen Dornen hat sich sehr wahrscheinlich mehrfach konvergent vollzogen. Beide Dornenformen können bei homologen Nesselkapseln, wie z. B. bei den kleinen Holotrichen II aus dem Scapus verschiedener Zoantharia (vgl. Abb. 8f, g) vorkommen. Haplonemen mit spitzen Dornen sind daher kein konstitutives Merkmal. Die zum Teil sehr spezifischen Dornen mancher Haplonemen z. B. der Madreporaria oder der Zoantharia sind dagegen autapomorphe Merkmale dieser Anthozoengruppen (Tab. 1).

\section{Heteronemen}

Das Kennzeichen der Heteronemen der Anthozoen besteht darin, daß sich die Bewaffnung, d. h. nicht nur die Größe, sondern auch die Form der Dornen unabhängig von Querschnittsänderungen des Schlauches abrupt und ohne Ubergang ändert. Teilweise ist der als Faden bezeichnete distale Schlauchteil oder ein als Faltstüdk bezeichneter proximaler Teil des Schaftes unbewaffnet (vgl. SснміDт 1969). Die Heteronemen der Anthozoen gehören ausschließlich zu den Rhabdoiden, deren Schaft weitgehend isodiametrisch ist (WEILI 1934). CARLGRENS (1940) Aufteilung der Rhabdoiden in bund p-Rhabdoiden hat sich insofern als sinnvoll erwiesen, als mit dieser Gliederung bei sämtlichen Hexacorallia rhabdoide Heteronemen mit unterschiedlicher Fadenbewaffnung gekennzeichnet werden. Die b-Rhabdoiden sind sämtlich hoplotel, d. h. sie haben einen bewaffneten Faden. Bei den Ceriantharia sind sie ausnahmslos mit T-förmigen Fadendornen ausgestattet, während die p-Rhabdoiden der Ceriantharia wie bei den Actiniaria und Zoantharia einen unbewaffneten Faden haben und daher anoplotel sind (Tab. 1). 
Bei den b-Rhabdoiden der Actiniaria kommen nur sehr selten T-förmige Fadendornen vor. Die meisten b-Rhabdoiden der Actiniaria haben wie die b-Rhabdoiden der Zoantharia und der Antipatharia kurze, spitze Fadendornen, die sich deutlich von den relativ langen, dünnen Fadendornen der p-Rhabdoiden der Antipatharien unterscheiden. Die kleinen deutlich verschiedenen rhabdoiden Heteronemen der Octocorallia, der Madreporaria und der Antipatharia lassen sich nicht in b- und p-Rhabdoiden aufgliedern. Da T-förmige Dornen bei den Haplonemen am ganzen Schlauch, bei den Heteronemen aber nur am Faden vorkommen, kann angenommen werden, daß die Anlage eines Schaftes mit seinen spezifischen Dornen apomorph entstanden ist.

\section{b-Rhabdoiden}

Für einen gemeinsamen Ursprung der b- und p-Rhabdoiden der Hexacorallia liefern die Ceriantharia die besten Hinweise. Die b- und $p$-Rhabdoiden der Ceriantharia zeigen keine Unterschiede in Form, Größe und Anordnung der Schaftdornen. Die b-Rhabdoiden der Ceriantharia zeichnen sich außerdem im Gegensatz zu denjenigen der übrigen Hexacorallia durch einen besonders breiten Schaft und eine besondere Größe aus. Dadurch ist bei den Ceriantharia, abgesehen von der Länge und Bewaffnung von Faden und Faltstück, der Unterschied zwischen b- und p-Rhabdoiden sehr gering. Bei den b-Rhabdoiden verhindert lediglich das mit kleinen Schaftdornen besetzte Ubergangsstück zwischen Schaft und Faden die Bildung einer trichterförmigen Offnung am Ende des nicht entladenen Schaftes (Abb. 2a, 3e). Wie bei keiner anderen Anthozoengruppe wird der gemeinsame Ursprung von $b$ - und $\mathrm{p}$-Rhabdoiden außerdem durch ein so einheitliches Vorkommen beider Nesselkapseltypen bewiesen. In sämtlichen Körperbereichen, in denen bei Ceriantboides $\mathrm{p}$-Rhabdoiden zu finden sind, sind bei Cerianthus bzw. Pachycerianthus annähernd form- und größengleiche bRhabdoiden zu finden. Ein kleiner Unterschied besteht lediglich darin, daß die b-Rhabdoiden II mit deutlichem langen Faltstück bei Pachyceriantbus in den Marginaltentakeln und die p-Rhabdoiden IIb bei Ceriantboides in den Labialtentakeln vorkommen. Bei der auffälligen Homologie der übrigen Rhabdoiden weist dieser Befund darauf hin, daß die Faltstücke bei $\mathrm{b}$ - und $\mathrm{p}$-Rhabdoiden konvergent entstanden sind. Dies trifft höchst wahrscheinlich auch für die großen b-Rhabdoiden in den Akontien von Metridium zu (vgl. SchMIDT 1969).

Bei allen übrigen Hexacorallia unterscheiden sich b- und p-Rhabdoiden deutlich an der Schaftbreite und -bewaffnung. Die mögliche Abstammung von den b-Rhabdoiden der Ceriantharia ist zumindest bei den Actiniaria zu erkennen, bei denen einige ursprüngliche Arten noch über b-Rhabdoiden mit T-förmigen Fadendornen verfügen. Als symplesiomorphes Merkmal haben sich die T-förmigen Fadendornen außer bei den b-Rhabdoiden der Ceriantharia auch bei den rhabdoiden Heteronemen I der Octocorallia und bei den speziellen b-Rhabdoiden der Protantheae und der Aiptasiadae („frühe“ Mesomyaria) sowie bei den p-Rhabdoiden der Madreporaria und Corallimorpharia erhalten (Tab. 1). Die b-Rhabdoiden mit spitzen Fadendornen sind demnach bei den Actiniaria, bei den Zoantharia und bei den Antipatharia und möglicherweise auch bei den Madreporaria und Corallimorpharia synapomorph (Tab. 1). 


\section{$p$-Rbabdoiden}

Wie oben erklärt wurde, ist der Unterschied zwischen b- und p-Rhabdoiden bei den Ceriantharia sehr gering. Das auffälligste Merkmal, in dem sich die p-Rhabdoiden von den b-Rhabdoiden unterscheiden, ist die deutliche Reduktion von Länge und Bewaffnung des Fadens. Dieses Merkmal ist eine bedeutende Synapomorphie sämtlicher p-Rhabdoiden nicht nur der Ceriantharia, sondern auch der Actiniaria und der Zoantharia. Die p-Rhabdoiden II der Ceriantharia und die p-Rhabdoiden B und C (vgl. SchмiD 1969) der Actiniaria sind außerdem in der Anlage von Faltstïcken relativ synapomorph. Die p-Rhabdoiden der Ceriantharia und die p-Rhabdoiden B der Actiniaria haben in entladenem Zustand die gleiche zur Kapsel hin gerichtete Dornenstellung. Sie stimmen außerdem bei den Ceriantharia und den ursprünglichen Actiniaria deutlich in Vorkommen und Größenverteilung überein. Die p-Rhabdoiden der Ceriantharia und die $p$ - $R$ habdoiden $B$ und $C$ der Actiniaria können daher in einer konstitutiven Gruppe zusammengefaßt werden, die hier vorläufig als B-Gruppe bezeichnet wird (Tab. 1).

Die p-Rhabdoiden A der Actiniaria und die p-Rhabdoiden der Zoantharia und mit Ausnahme der Länge und Bewaffnung des Fadens auch der Antipatharia sind in Form und Vorkommen völlig identisch und daher als homolog zu betrachten. Sie kommen stets zahlreich in den Mesenterialfilamenten, teilweise im Pharynx und sehr selten im Scapus und in den Tentaklen vor. Sie sind die einzigen p-Rhabdoiden der Zoantharia und der Antipatharia sowie fast sämtlicher Endomyaria, von denen nur noch wenige Arten über sehr kleine p-Rhabdoiden B verfügen (Tab. 1, vgl. Schmor 1972b). Die relativ langen spitzen Fadendornen der p-Rhabdoiden der Antipatharia sind daher entweder konvergent und damit autopomorph entstanden oder plesiomorph. Da sich die p-Rhabdoiden A der Actiniaria und die p-Rhabdoiden der Zoantharia und Antipatharia auch in anderen Merkmalen wie Dornenanordnung, Phasenkontrast und Kapselstruktur gleichen und von den p-Rhabdoiden der B-Gruppe unterscheiden, werden sie hier vorläufig in der konstitutiven A-Gruppe vereint (Tab. 1). A- und B-Gruppe können zu einer konstitutiven höheren Einheit zusammengefaßt werden, da sie gegenüber den $\mathrm{p}$-Rhabdoiden mit T-förmigen Fadendornen synapomorph sind.

Die p-Rhabdoiden der Madreporaira und der Corallimorpharia gleichen in der Dornenstellung den $\mathrm{p}$-Rhabdoiden der A-Gruppe, in der Dichte der Dornenwindungen aber mehr den p-Rhabdoiden der B-Gruppe, deren sie auch in Größe und Verteilung mehr entsprechen. In dem wichtigen Merkmal der Länge und Bewaffnung des Fadens sind sie aber symplesiomorph besonders mit den b-Rhabdoiden der Ceriantharien und ursprünglichen Aktinien (Tab. 1). Eine Ableitung der p-Rhabdoiden der Madreporaria ist daher zur Zeit nicht möglich.

Wie die Untersuchung ergeben hat, ist der morphologische Differenzierungsgrad der Nesselkapseln bei den einzelnen Anthozoengruppen sehr unterschiedlich. Eine Entwicklungstendenz von einfachen zu höher differenzierten Nesselkapseln ist nicht eindeutig zu erkennen. Die Atrichen sind interessanterweise gerade auf diejenigen Gruppen beschränkt, die andererseits die morphologisch am höchsten differenzierten Nesselkapseln aufweisen, nämlich auf die Ceriantharia und die Actiniaria. Dieser Befund deutet darauf hin, daß sich Haplonemen und Heteronemen unterschiedlich entwickelt 
haben und sehr wahrscheinlich unterschiedliche Funktionen haben. Die Haplonemen haben bei den Madreporaria und Corallimorpharia und in konvergenter Entwicklung bei den Zoantharia ihren höchsten Differenzierungsgrad erreicht. Sie haben dabei wesentlich kompliziertere Dornen entwickelt als die Heteronemen der Anthozoen. Die Heteronemen der Madreporaria, Corallimorpharia und Zoantharia weisen dagegen keine besonderen Differenzierungen auf, wie sie z. B. in Form von Faltstücken bei den Ceriantharia und Actiniaria zu beobachten sind. Diese beiden Anthozoengruppen weisen allein atriche Haplonemen auf und haben über einfache spitze Dornen hinaus keine auffällige Bewaffnung bei den Haplonemen hervorgebracht. Die Octocorallia haben recht komplizierte Heteronemen, aber keine Haplonemen entwickelt und weisen damit auf einen möglichen gemeinsamen Ursprung mit den Ceriantharia und Actiniaria hin.

\section{Die Bedeutung der Nesselkapseln für die phylogenetische Systematik der Anthozoen}

Wie eingangs erläutert wurde, ist das Ziel der phylogenetisch-systematischen Forschung, den monophyletischen Ursprung einer Artengruppe nachzuweisen bzw. monophyletische Arten in höheren systematischen Kategorien zu vereinen. Die Frage nach dem Ursprung einer Artengruppe ist daher gleichbedeutend mit der Frage nach der sogenannten Schwestergruppe, mit der sie allein auf eine gemeinsame Stammart zurückzuführen ist. Eine Artengruppe ist nur dann monophyletisch, wenn sie alle Nachkommen einer bestimmten Stammart enthält. Die Ergebnisse resultieren daher in einem Stammbaum, der rein dichotom aufgebaut ist. Der hier grob dargestellte Stammbaum der Anthozoen. (Abb. 10) zeigt, daß von den zur Zeit gültigen Ordnungen höchstens die Antipatharia eine monophyletische Gruppe darstellen. Die „späten“ Zoantharia sind es schon nicht mehr, da auch sämtliche Antipatharia Nachkommen ihrer Stammart sind. Der Stammbaum der Anthozoen ist weit komplexer als hier dargestellt. Um sämtliche Teilungsschritte aufzuzeigen, wäre die Untersuchung aller Anthozoenarten erforderlich. Hier werden lediglich mit Ausnahme der Antipatharia die Ordnungen der Hexacorallia in verschiedene Gruppen aufgeteilt, um den komplexen Charakter des Anthozoenstammbaumes anzudeuten.

Die Frage nach dem gemeinsamen Ursprung der Octo- und Hexacorallia kann vorläufig nicht mit Sicherheit beantwortet werden, da der Ursprung der Anthozoen noch unbekannt ist. Es kann daher nur vermutet werden, daß die rhabdoiden Heteronemen mit T-förmigen Dornen möglicherweise ein relativ synapomorphes Merkmal der Octo- und Hexacorallia sind. Eindeutig synapomorphe Merkmale fehlen. Entgegen der bisherigen Auffassung (WeILI 1934, CARLGREN 1940, Werner 1965) besteht das Monocnidom der Octocorallia nicht aus dem morphologisch einfachsten (atriche Haplonemen), sondern aus dem kompliziertesten Nesselkapseltyp, der bei Anthozoen vorkommt. Sollten Octo- und Hexacorallia auf eine gemeinsame Stammart zurückgeführt werden können, so ist festzuhalten, daß innerhalb der phylogenetischen Entwicklung der Anthozoen die Ausbildung komplizierter Nesselkapseln ein plesiomorphes Merkmal ist. Die Spirocysten sind dagegen ein synapomorphes Merkmal der Hexacorallia.

Von zahlreichen Autoren (zuletzt Hyman 1940, Welds \& Hill 1956, ReNzi 1966) werden Ceriantharia und Antipatharia als Ceriantipatharia zusammengefaßt, weil sie 
einen gemeinsamen Ursprung haben sollen. Die Vereinigung dieser Anthozoenordnungen ist aber völlig unberechtigt, wie bereits VAN PeSCH (1914) verdeutlichte und wie mit den vorliegenden Befunden bewiesen wird. Der gemeinsame Ursprung wird hauptsächlich mit der vorrangigen Ausbildung von Transversalsepten bei Antipatharia und Ceriantharienlarven begründet (z. B. Hyman 1940). Die gleiche Entwicklung ist aber auch bei anderen Anthozoen, z. B. bei den Zoantharia zu beobachten, wie von HYMAN (1940, Abb. $204 \mathrm{H}-\mathrm{K}$ ) selbst dargestellt wird. Es handelt sich allenfalls, wie bei den meisten entwicklungsgeschichtlichen Merkmalen, um ein symplesiomorphes Merkmal. Nach HaND (1966) sollen sich dagegen Ceriantharia und Antipatharia ebenso isoliert gegenüberstehen wie die übrigen Hexacorallia und die Octocorallia.

Die vorliegende Untersuchung hat ergeben, daß die Ceriantharia die ursprünglichsten Hexacorallia und die Schwestergruppe zumindest der Actiniaria sind. Als ursprüngliche Merkmale ist die große Übereinstimmung von Atrichen und Holotrichen einerseits und $b$ - und $p$-Rhabdoiden andererseits zu werten. Letztere weisen auf eine frühere einheitliche Rhabdoidenform hin, wie sie bei den Octocorallia vorkommt. Absolut plesiomorph sind die Ceriantharia in der Bewaffnung von Holotrichen und bRhabdoidenfäden, die ausschließlich aus T-förmigen Dornen besteht. Die Ausbildung komplizierter rhabdoider Heteronemen und die atrichen Haplonemen sind symplesiomorphe Merkmale der Ceriantharia und Actiniaria. Außer diesen Merkmalen sind die ektodermale Längsmuskulatur und die fehlende Basilarmuskulatur symplesiomorphe Merkmale der Ceriantharia und der meisten ursprünglichen Actiniaria (Tab. 1). Als ein besonders bemerkenswertes symplesiomorphes Merkmal beider Anthozoenordnungen ist das identische Schwimmverhalten zu werten, das RoBSON $(1966,1971)$ allerdings in Unkenntnis der phylogenetischen Zusammenhänge bei Ceriantharia und einigen nunmehr als ursprünglich erkannten Actiniaria (ScHmid 1972b) beschrieben hat (Tab. 1). Das bei Gonactinia (Protantheae), Boloceroides, Bunodeopsis (beide Boloceroidaria) und Ceriantharia völlig identische Schwimmverhalten ist bei Berücksichtigung der vorliegenden Befunde nicht mehr als Konvergenz zu bewerten. Ein ganz anderes Schwimmverhalten beobachtete RoBson (1966) bei Stompbia coccinea, die $\mathrm{zu}$ den "späten“ Mesomyaria gehört. Dieses Schwimmverhalten ist dagegen konvergent entwickelt worden und daher ein autapomorphes Merkmal. Die bisher aufgefürten Merkmale sind zwar symplesiomorph, aber nicht konstitutiv. Daß Ceriantharia und Actiniaria Schwestergruppen sind, geht dagegen eindeutig aus den anoplotelen p-Rhabdoiden und insbesondere aus den p-Rhabdoiden der B-Gruppe hervor (Tab. 1). Diese Nesselkapseln sind, wie oben gezeigt wurde, nach Form und Vorkommen besonders auffällige relativ synapomorphe Merkmale beider Anthozoenordnungen.

Innerhalb der Anthozoen haben die Actiniaria die mannigfaltigsten Formen hervorgebracht, was nicht zuletzt in den zahlreichen Nesselkapseltypen zum Ausdruck kommt (SсHмidт 1969). Im Verlauf der phylogenetischen Entwicklung der Actiniaria (vgl. Schmid 1972b) werden die p-Rhabdoiden in Form und Vorkommen zu den relativ einfach gebauten und meist nur auf die Mesenterialfilamente beschränkten p-Rhabdoiden A reduziert. Die ursprünglichen Actiniaria (Protantheae, Boloceroidaria, "frühe" Mesomyaria) haben als plesiomorphe Merkmale Reste von ektodermaler Längsmuskulatur im Scapus, spitzköpfige Spermien und keine p-Rhabdoiden A, sondern nur $\mathrm{p}-\mathrm{R}$ habdoiden der B-Gruppe, die in allen Körperbereichen vorkommen. Die 


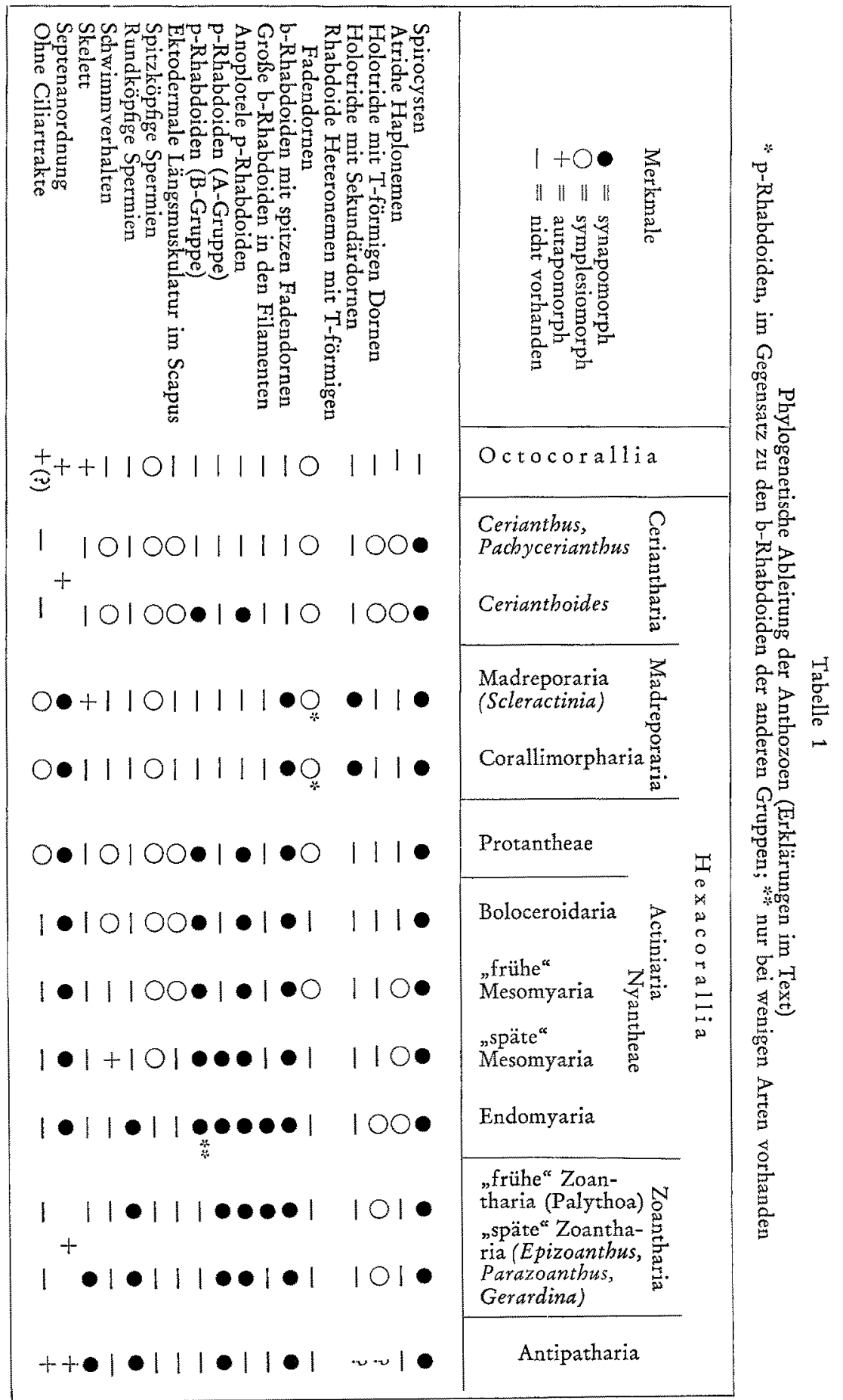




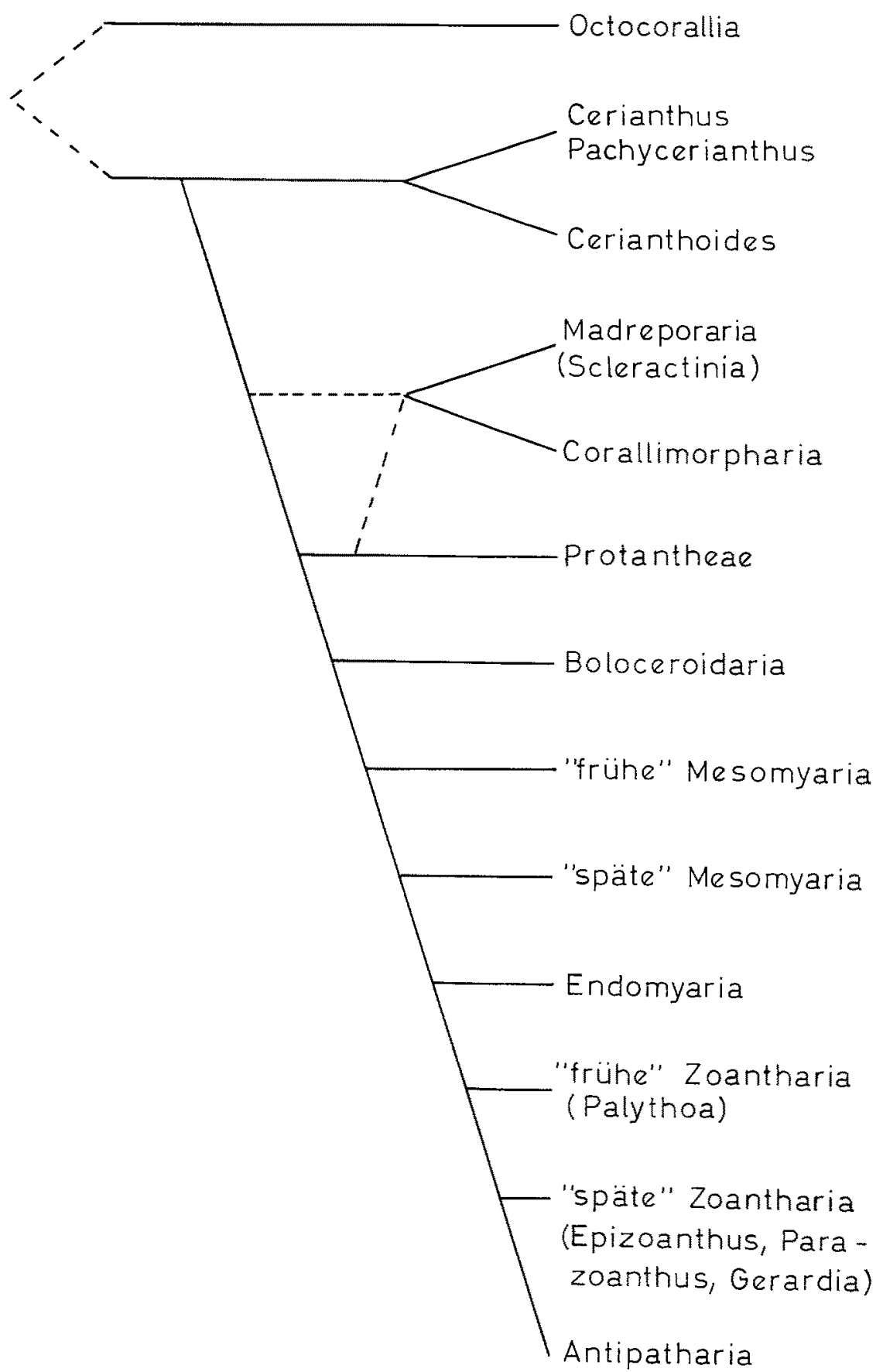

Abb, 10: Stammbaum der Anthozoa 
abgeleiteten Actiniaria (Endomyaria) haben als apomorphe Merkmale keine ektodermale Längsmuskulatur im Scapus, rundköpfige Spermien, mit wenigen Ausnahmen ausschließlich p-Rhabdoiden A und große b-Rhabdoiden in den Mesenterialfilamenten (Tab. 1). Bei den Endomyaria kommen nur noch bei wenigen Arten p-Rhabdoiden B in den Mesenterialfilamenten vor. Beide Gruppen werden durch zahlreiche "späte“ Mesomyaria verbunden, die als plesiomorphe Merkmale noch spitzköpfige Spermien, p-Rhabdoiden $B$ in fast allen Körperbereichen und keine großen b-Rhabdoiden in den Mesenterialfilamenten haben. Sie sind aber in der fehlenden ektodermalen Längsmuskulatur und in den p-Rhabdoiden A synapomorph mit den Endomyaria (Tab. 1). Die klassische Auffassung, wonach Aktinien mit nur 8 vollständigen Septen (sogen. $E d$ wardsia-Stadium) als ursprünglich gelten, da sich dieses Merkmal von den Octocorallia ableiten soll, ist mit den vorliegenden Befunden widerlegt. Protantheae und Edwardsia galten auf Grund ihrer 8 vollständigen Septen als die ursprünglichsten Actiniaria. Auf Grund ihres Cnidoms gehört aber die Gattung Edwardsia im Gegensatz zu den tatsächlich ursprünglichen Protantheae zu den phylogenetisch "jüngsten" Actiniaria, den Endomyaria.

Zweifellos resultiert die phylogenetische Entwicklung der Actiniaria über die Endomyaria hinaus in der Stammart von Zoantharia und Antipatharia. Die Endomyaria weisen zahlreiche Synapomorphien mit diesen Ordnungen, besonders mit den „frühen" Zoantharia auf. Mit Ausnahme der Haplonemen und der kleinen b-Rhabdoiden und p-Rhabdoiden $B$ in den Mesenterialfilamenten stimmen Endomyaria und "frühe" Zoantharia in Form und Vorkommen der übrigen Nesselkapseln weitgehend überein. Das Fehlen der kleinen b-Rhabdoiden in den Mesenterialfilamenten und die speziellen b-Rhabdoiden im Scapus sind apomorphe Merkmale der Zoantharia. Die bei sämtlichen Actiniaria und Zoantharia morphologisch absolut identischen p-Rhabdoiden der A-Gruppe kommen fast ausschließlich in den Mesenterialfilamenten und teilweise im Pharynx vor. Nur einige "frühe" Zoantharia, Endomyaria und Antipatharia haben sie auch im Scapus und in den Tentakeln. Ein besonderes relativ synapomorphes Merkmal, die großen b-Rhabdoiden in den Mesenterialfilamenten, verbindet Endomyaria und „frühe" Zoantharia. Die Palythoa sind demnach die ursprünglichsten Zoantharia, und die fehlenden großen b-Rhabdoiden ein synapomorphes Merkmal der „späten" Zoantharia und der Antipatharia. Ein bedeutendes synapomorphes Merkmal der Endomyaria, der Zoantharia und der Antipatharia sind schließlich die rundköoffigen Spermien (Tab. 1). Zu den „späten "Zoantharia gehört auf Grund der vorliegenden Nesselkapselbefunde $\mathfrak{u}$, a. auch Gerardia savaglia. Diese Art zeichnet sich gegenüber sämtlichen anderen Zoantharia durch ein äußerst festes, den Gorgonien äußerlich weitgehend ähnliches Skelett aus. Die Vermutung, daß das Skelett von Gerardia nicht von der Zoantharie, sondern von einer abgestorbenen Gorgonie stammt, haben Roche \& TIXIER-DurIvault (1951) widerlegt. Die Autoren konnten nachweisen, daß das Skelett von Gerardia sich biochemisch erheblich von dem der Gorgonaria unterscheidet und in dieser Hinsicht mit der Skelettsubstanz der Antipatharia ibereinstimmt. In Wuchsform und OKologie bestehen ebenfalls deutliche Unterschiede zwischen Gerardia und Gorgonaria (ScHMIDT 1972a). Dieser bemerkenswerte Skelettbefund ist ein sicheres synapomorphes Merkmal der Zoantharia und der Antipatharia (Tab. 1). 
Wie oben gezeigt wurde, gehören die p-Rhabdoiden der Antipatharia zur AGruppe, da sie bis auf die Länge und Bewaffnung des Fadens in Form und Vorkommen mit den $\mathrm{p}$-Rhabdoiden A der Actiniaria und der Zoantharia übereinstimmen. Die spitzen Fadendornen können plesiomorph oder als autapomorphes Merkmal konvergent entwickelt worden sein. Nach Trschererek (1936) sollen die bei den Antipatharia selten vorkommenden Holotrichen denjenigen der Zoantharia in Form und Vorkommen (bis auf die Mesenterialfilamente) gleichen. Die Ableitung der Antipatharia zeigt, daß diese Anthozoengruppe im Gegensatz zu der Auffassung vaN PEsch's (1914) im Verlauf der Phylogenie der Anthozoen morphologisch stark reduziert wurde. Gonaden und Filamente, die außer an den Transversalsepten auch an anderen Septen gefunden wurden, sind daher plesiomorphe Merkmale. Zu dieser Ansicht gelangt auch CARLGREN (1924), der bei Palaeozoanthus sogenannte fertile Mikrosepten, die gewöhnlich keine Gonaden haben, beobachtete. Die morphologische Reduktion insbesondere der Septen beginnt also bereits bei den Zoantharia und kann daher als synapomorphes Merkmal der Zoantharia und der Antipatharia betrachtet werden. Die morphologische Reduktion der Antipatharia ist allerdings nicht als Degeneration aufzufassen, sondern hängt eng mit der Lebensweise auf relativ dünnen und häufig starker Strömung ausgesetzten Skeletten zusammen.

Die Nesselkapselbefunde der Madreporaria haben nur wenige Hinweise auf verwandtschaftiche Beziehungen zu anderen Anthozoen ergeben. Zu den Madreporaria werden hier auch die Corallimorpharia gezählt. Diese früher als stichodactyline Aktinien bezeichneten skelettlosen Anthozoen stimmen mit Ausnahme der kleineren Holotrichen II in Form und Vorkommen aller übrigen Nesselkapseltypen mit den Madreporaria überein. CARLGREN $(1940,1949)$ hat sie daher als Corallimorpharia neben die anderen Anthozoenordnungen gestellt. Die vorliegende Untersuchung hat ergeben, daß die Identität der Nesselkapseln so groß ist, daß die Corallimorpharia besser innerhalb der Madreporaria neben die eigentlichen skelettbildenden Madreporaria, die Scleractinia, zu stellen sind (Abb. 10). Die Holotrichen I sind durch die Ausbildung von Sekundärdornen ein sehr markantes synapomorphes Merkmal der Corallimorpharia und der Scleractinia (vgl. Abb. 6a, b, 9a, b, Tab. 1). Die Holotrichen II der Corallimorpharia, die am proximalen Schlauchteil atrich sind, könnten eventuell symplesiomorph mit den Atrichen der Actiniaria und der Ceriantharia sein. Besondere konstitutive Merkmale sind aber bei den Nesselkapseln der Actiniaria und der Corallimorpharia nicht festzustellen. HAND (1966) begründet die Abstammung der Actiniaria von den Scleractinia u. a. mit übereinstimmenden Nesselkapseln. Nach seiner Auffassung sollen die pRhabdoiden und die Holotrichen I, die die einzigen Holotrichen der Scleractinien sind, mit den p-Rhabdoiden und mit den speziellen Akrorhagenhaplonemen der Endomyaria übereinstimmen, die HAND als die ursprünglichsten Actiniaria betrachtet. Diese Behauptung wird mit den vorliegenden Bedunden widerlegt. Besonders die erwähnten Haplonemen unterscheiden sich kraß, da sie über die höchstentwickelten bzw. über die ursprünglichsten Dornen verfügen. Von den Nesselkapseln der Actiniaria und der Madreporaria sind nur die b-Rhabdoiden mit spitzen Fadendornen als synapomorphes Merkmal zu werten (Tab. 1). Der schwache Nesselkapselbefund der Madreporaria ist um so bemerkenswerter, als diese Anthozoengruppe auf Grund der gleichartigen Septenanordnung als besonders nah verwandt mit den Actiniaria gilt (u. a. Hyman 1940, 
Wells \& Hill 1956, Hand 1966, Renzi 1966). Die gleichartige Septenanordnung ist somit ein weiteres Merkmal, das bei Actiniaria und Madreporaria als synapomorph zu bezeichnen ist. Auf den gemeinsamen Ursprung weisen möglicherweise auch die fehlenden Ciliartrakte (Tab. 1) und die speziellen b-Rhabdoiden der ursprünglichen Actiniaria hin, die bei den Protantheae außer T-förmigen Fadendornen einen sehr kurzen Schaft wie die meisten Rhabdoiden, speziell in den Tentakeln, der Madreporaria haben. Der Ursprung der Madreporaria kann mit den vorliegenden Merkmalen nicht mit Sicherheit bestimmt werden. Sie sind entweder die Schwestergruppe der Actiniaria und somit zusammen mit diesen die Schwestergruppe der Ceriantharia, oder sie leiten sich von den ursprünglichen Actiniaria, z. B. den Protantheae, ab (Abb. 10).

\section{ZUSAMMENFASSUNG}

1. Phasen- und elektronenoptische Untersuchungen an Nesselkapseln von 35 Anthozoenarten aus Atlantik, Mittelmeer und Rotem Meer haben bei den einzelnen Klassen und Ordnungen eine große morphologische Divergenz der bisher gültigen Nesselkapseltypen (WeIll 1934, CARLgren 1940) ergeben. Weills Nesselkapseltypen haben daher nur geringe taxonomische und phylogenetische Bedeutung.

2. Atriche Haplonemen kommen nur bei den Ceriantharia und Actiniaria vor. Sie sind symplesiomorph.

3. Die holotrichen Haplonemen weisen die größte Dornenmannigfaltigkeit auf. Die Dornen sind teilweise morphologisch wesentlich differenzierter als bei den rhabdoiden Heteronemen. Die meisten holotrichen Haplonemen sind auf Grund ihrer speziellen Dornenstruktur apomorphe Merkmale verschiedener Anthozoengruppen. Sie sind aber als Kategorie kein konstitutives Merkmal.

4. Als symplesiomorphes Merkmal kommen bei zahlreichen Anthozoen am Schlauch verschiedener holotricher Haplonemen und am Faden verschiedener rhabdoider Heteronemen T-förmige Dornen vor. Spitze Dornen oder unbewaffnete Endfäden sind apomorphe Merkmale.

5. Rhabdoide Heteronemen sind der einzige Nesselkapseltyp der Octocorallia, die sich wie die b-und p-Rhabdoiden der Hexacorallia in der Bewaffnung des Fadens unterscheiden.

6. Auf Grund der vorliegenden Nesselkapselbefunde sind die Ceriantharia die ursprünglichsten Hexacorallia. Die Ceriantharia verfügen wie die ursprünglichen Actiniaria über die einfachsten Haplonemen und die am höchsten differenzierten Heteronemen.

7. Actiniaria und Zoantharia sind in der Ausbildung anoploteler p-Rhabdoiden synapomorph mit einigen Ceriantharia.

8. Die Zoantharia sind mit den Actiniaria in der Ausbildung völlig identischer pRhabdoiden ( $\mathrm{p}$-Rhabdoiden A) synapomorph.

9. Als Schwestergruppe der Zoantharia sind die Antipatharia zu betrachten, die über weitgehend gleiche rhabdoide Heteronemen, mit Ausnahme einiger autapomorpher Typen, wie die Zoantharia verfügen. 
10. Die Nesselkapseln der Madreporaria (Scleractinia) und der Corallimorpharia stimmen mit Ausnahme einiger nur den Corallimorpharia eigenen spezieller Holotrichen in allen morphologischen Details vollständig überein. Die Nesselkapselbefunde beider Anthozoengruppen, die hier einheitlich als Madreporaria zusammengefaßt werden, haben dagegen nur wenige Hinweise auf verwandtschaftliche Beziehungen $z \mathfrak{u}$ den Actiniaria ergeben. Die Ergebnisse werden in einer Stammbaumtabelle dargestellt.

Danksagungen. Fräulein G. DeichgräBER und Herrn G. Dafnis danke ich sehr herzlich fïr Rat und Hilfe bei den elektronenoptischen Untersuchungen. Den meeresbiologischen Star tionen von Neapel und Eilat danke ich für die Arbeitsmöglichkeiten. Herrn Dr. T. LunDäLv und der Station von Kristineberg gilt mein besonderer Dank für die Obersendung von Lebendniaterial. Die Arbeit wurde mit dankenswerter Förderung durch die Deutsche Forschungsgemeinschaft ausgeführt.

\section{ZITIERTE LITERATUR}

Carlgren, O., 1924. Die Larven der Ceriantharien, Zoantharien und Actiniarien mit einem Anhange zu der Zoantharia. Wiss. Ergebn. dt. Tiefsee-Exped. "Valdivia", 19 (8), 341-476.

- 1937. Ceriantharia and Zoantharia. Rep. G. Barrier Reef Exped. 1928-29. 5 (5), 177-207.

- 1938. South African Actiniaria and Zoantharia. K. svenska VetenskAkad. Handl. 17 (3), $1-148$.

- 1940. A contribution to the knowledge of the structure and distribution of the cnidae in the Anthozoa. Acta Univ. lund. N. F. (Avd. 2) 36 (3), 1-62.

- 1945. Further contribution to the knowledge of the cnidom in the Anthozoa, especially in the Actiniaria. Acta Univ. lund. N. F. (Avd. 2) 41 (9), 1-24.

- 1949. A survey of the Ptychodactiaria, Corallimorpharia and Actiniaria. K. svenska VetenskAkad. Handl. 1 (1), 1-121.

Curress, C. E., 1955. An interpretation of the structure and distribution of cnidae in Anthozoa. Syst. Zool. 4, 120-137.

Dantan, J. L., 1921. Recherches sur les Antipathaires. Archs. Anat. microse. 17, 137-237.

Doumenc, D., 1971. Aspects morphologiques de la devagination du spirocyste chez Actinia equina L. J. Microsc. 12, 263-270.

Hand, C., 1961. Present state of nematocyst research: Types, structure and function. In: Biology of Hydra and some other coelenterates. Ed. by H. M. Lenнoff \& W. F. Loomis. Univ. Miami Press, Coral Gables, 187-202.

- 1966. On the evolution of the Actiniaria. In: The Cnidaria and their evolution. Ed. by, W. J. Rezs. Acad. Press, London, 135-146. (Symp. zool. Soc. Lond. 16.)

HenNig, W., 1969. Die Stammesgeschichte der Insekten. Kramer, Frankfurt, 436 pp.

Hyman, L. H., 1940. The Invertebrates. Bd 1: Protozoa through Centophora. McGraw-Hill, New York, 726 pp.

KaestNer, A., 1969. Lehrbuch der speziellen Zoologie. - Fischer, Stuttgart, 1 (1), 1-898. $1-898$.

Pesch A. J., van, 1914. The Antipatharia of the Siboga Expedition. Siboga Exped. 17, 1-258.

Picken \& SkAer, R. J., 1966. A review of researches on nematocysts. In: The Cnidaria and their evolution. Ed. by W. J. ReEs. Acad. Press, London, 19-50. (Symp. zool. Soc. Lond. 16.)

RENZI, M., DE, 1966. Sobre la filogenia de los cnidarios particularmente de la clase de los antozoos. Instituto Biol. apl. Barcelona 41, 89-101.

Rosson, E. A., 1953. Nematocysts of Corynactis: The activity of the filament during discharge. Q. Il microsc. Sci. 94, 229-235. 
- 1966. Swimming in Actiniaria. In: The Cnidaria and their evolution. Ed. by W. J. ReEs. Acad. Press, London, 333-360. (Symp. zool. Soc. London 16)

- 1971. The behaviour and neuromuscular system of Gonactinia prolifera, a swimming seaanemone. J. exp. Biol. 55, 611-640.

Roche, J. \& Trxier-Durivault A., 1951. Rapports des Gerardiides avec les Zoanthides et les Antipathaires. Bull. Mus. natn. Hist. nat., Paris 23, 402-409.

Sснмдт, H., 1969. Die Nesselkapseln der Aktinien und ihre differentialdiagnostische Bedeutung. Helgoländer wiss. Meeresunters. 19, 284-317.

- 1972a. Bionomische Studien an mediterranen Anthozoen: die Anthozoenfauna des Strombolicchio (Aolische Inseln). Mar. Biol. 15, 265-278.

- 1972b. Prodromus zu einer Monographie der mediterranen Aktinien. Zoologica, Stuttgart 121.

- \& Béress, L., 1971. Phylogenetische Betrachtungen zur Toxizität und Nesselwirkung einiger Actiniaria (Anthozoa) im Vergleich zur Morphologie ihrer Nesselkapseln. Kieler Meeresforsch. 27, 166-170.

Seifert, R., 1928. Die Nesselkapseln der Zoantharien und ihre differentialdiagnostische Bedeutung. Zool. Jb. (Syst. Okol. Geogr. Tiere) 55, 419-500.

Skaer, R. J. \& Picken, L. E. R., 1965. The structure of the nematocyst thread and the geometry of discharge in Corynactis viridis Allman Phil. Trans. R. Soc. (B) 250 (764), 131-164.

StEPheNson, T. A., 1929. On the nematocysts of sea anemones. J. mar. biol. Ass. U. K. 16, 173-201.

TIschbIEREK, H., 1936. Die Nesselkapseln der Antipatharien und ihre differentialdiagnostische Bedeutung. Diss. Breslau, 60 pp.

WEILL, R., 1934. Contribution a l'étude des Cnidaires et leur nématocystes. II. valeur taxonomique du cnidom. Trav. Stn. zool. Wimereux 10-11, 1-701.

Wells, J. W. \& HrLl, D., 1956. Anthozoa-General features. In: Treatise on invertebrate paleontology. P. F: Coelenterata. Ed. by R. C. Moore. Geol. Soc. America, New York, 161-165.

Werner, B., 1965. Die Nesselkapseln der Cnidaria, mit besonderer Berücksichtigung der Hydroida. I. Klassifikation und Bedeutung für die Systematik und Evolution. Helgoländer wiss. Meeresunters. 12, 1-39.

WESTFAlL, J. A., 1962. Fine structure of nematocysts in a sea anemone. Int. Conf. Electron Microsc. 5, $\mathrm{M}-13$.

WestFald, J. A., 1965. Nematocysts of the sea anemone Metridium. Am. Zool. 5, 377-393.

WiLL, L., 1909. Die Klebkapseln der Aktinien und der Mechanismus ihrer Entladung. Sber. Abh. naturf. Ges. Rostock 1, 65-103.

Anschrift des Autors: Dr. Dr. Hajo SchmidT

Zoologisches Institut der Universität

69 Heidelberg

Berliner Straße 15

Bundesrepublik Deutschland 